\title{
The Circumgalactic Medium in Massive Halos
}

\author{
Hsiao-Wen Chen
}

\begin{abstract}
This chapter presents a review of the current state of knowledge on the $\operatorname{cool}\left(T \sim 10^{4} \mathrm{~K}\right)$ halo gas content around massive galaxies at $z \approx 0.2-2$. Over the last decade, significant progress has been made in characterizing the cool circumgalactic gas in massive halos of $M_{h} \approx 10^{12-14} \mathrm{M}_{\odot}$ at intermediate redshifts using absorption spectroscopy. Systematic studies of halo gas around massive galaxies beyond the nearby universe are made possible by large spectroscopic samples of galaxies and quasars in public archives. In addition to accurate and precise constraints for the incidence of cool gas in massive halos, detailed characterizations of gas kinematics and chemical compositions around massive quiescent galaxies at $z \approx 0.5$ have also been obtained. Combining all available measurements shows that infalling clouds from external sources are likely the primary source of cool gas detected at $d_{\sim} 100 \mathrm{kpc}$ from massive quiescent galaxies. The origin of the gas closer in is currently less certain, but SNe Ia driven winds appear to contribute significantly

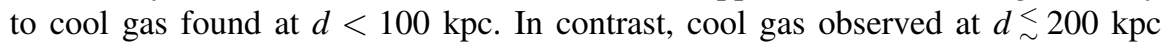
from luminous quasars appears to be intimately connected to quasar activities on parsec scales. The observed strong correlation between cool gas covering fraction in quasar host halos and quasar bolometric luminosity remains a puzzle. Combining absorption-line studies with spatially-resolved emission measurements of both gas and galaxies is the necessary next step to address remaining questions.
\end{abstract}

\section{Introduction}

In the standard picture of galaxy formation and evolution, primordial gas first cools and condenses within dark matter halos to form stars (e.g. White \& Rees, 1978; Blumenthal et al., 1984). Gas in high-mass halos with dark matter halo mass exceeding

Hsiao-Wen Chen

Department of Astronomy \& Astrophysics, and Kavli Institute for Cosmological Physics, The University of Chicago, Chicago, IL 60637, USA, e-mail: hchen@ odd job . uchicago . edu 
$M_{h} \approx 10^{12} \mathrm{M}_{\odot}$ is expected to be shock-heated to high temperatures (e.g. Birnboim \& Dekel, 2003; Kereš et al., 2005, 2009; Dekel \& Bernboim, 2006). Within the hot gas halos, thermal instabilities can induce the formation of pressure-supported cool clouds (e.g. Mo \& Miralda-Escudé, 1996; Maller \& Bullock, 2004; Sharma et al., 2012; Voit et al., 2015). In lower mass halos, cool filaments from the intergalactic medium can reach deep into the center of the halo without being shock-heated. Both condensed cool clouds of hot halos and cool filaments can, in principle, supply the fuels necessary to sustain star formation in galaxies. As new stars form and evolve, the surrounding interstellar and circumstellar gas is expected to be heated and enriched by heavy elements ejected from massive stars, regulating subsequent star formation.

Several semi-analytic studies have been carried out in searching for a general prescription that connects galaxies found in observations to dark matter halos formed in theoretical frameworks. These studies seek to establish a mean relation between galaxy stellar mass $\left(M_{\text {star }}\right)$ and host halo mass $\left(M_{h}\right)$ by matching the observed space density of galaxies with the expected abundance of dark matter halos as a function of mass (e.g. Vale \& Ostriker, 2004; Shankar et al., 2006). The mean galaxy mass and halo mass ratio is found to peak at $M_{h} \sim 10^{12} \mathrm{M}_{\odot}$ with $M_{\text {star }} / M_{h} \approx 0.04$ and declines rapidly both toward higher and lower masses (e.g. Moster et al., 2010; Guo et al., 2010; Behroozi et al., 2010). The declining $M_{\text {star }} / M_{h}$ indicates a reduced star formation efficiency in both low- and high-mass halos. Different feedback mechanisms are invoked in theoretical models in order to match the observed low star formation efficiency in low- and high-mass halos. As supernova-driven winds are thought to suppress star formation efficiency in low-mass dwarf galaxies (e.g. Larson, 1974; Dekel \& Silk, 1986), feedback due to active galactic nuclei (AGN) powered by supermassive black holes is invoked to quench star formation in high-mass halos, resulting in massive quiescent galaxies (e.g. Bower et al., 2006; Croton et al., 2006; Dubois et al., 2013). While blueshifted broad absorption and emission lines are commonly seen in luminous quasars, indicating the presence of high-speed outflows, direct observational evidence of AGN feedback on large scales $(\sim 10-100$ kpc) remains scarce (e.g. Alexander et al., 2010; Greene et al., 2012; Maiolino et al., 2012) and see also Fabian (2012) for a review.

Observations of the circumgalactic medium (CGM) in massive halos offer complementary and critical constraints for the extent of feedback and gas accretion (e.g. Somerville \& Davé, 2015). In particular, the circumgalactic space within the halo radius, $R_{\mathrm{vir}}$, lies between galaxies, where star formation takes place, and the intergalactic medium (IGM), where 90 percent of all baryonic matter in the universe resides (Rauch et al., 1997). As a result, CGM properties are shaped by the complex interactions between IGM accretion and outflows driven by energetic feedback processes in the galaxies.

Imaging observations of the cool $\left(T \sim 10^{4} \mathrm{~K}\right)$ CGM are only feasible in $21 \mathrm{~cm}$ surveys at $z \lesssim 0.2$, because, with few exceptions (e.g. Cantalupo et al., 2014; Fernández et al., 2016; Hayes et al., 2016), the gas density is typically too low to be detected in emission. Absorption-line spectroscopy of background quasars provides a powerful, alternative tool for studying this tenuous gas in the distant universe 
based on the absorption features imprinted in the quasar spectra (e.g. Lanzetta et al., 1995; Bowen et al., 1995; Steidel et al., 2002). But, because both quasars and massive galaxies are rare, close pairs of massive galaxies and background quasars by chance projection are even rarer. Studying the CGM around massive galaxies using quasar absorption spectroscopy therefore requires a large spectroscopic sample of galaxies and quasars over a substantial volume in order to assemble a statistical sample of massive galaxy and quasar pairs. The Sloan Digital Sky Survey (SDSS; York et al., 2000) has produced a large spectroscopic archive of distant galaxies and quasars, facilitating the assembly of a statistically significant sample of close massive galaxy and quasar pairs, as well as a large sample of projected quasar pairs. These pair samples have enabled systematic studies of halo gas beyond the nearby universe using absorption spectroscopy.

This chapter presents a review of the current state of knowledge on the cool CGM properties in massive halos of $M_{h} \approx 10^{12-14} \mathrm{M}_{\odot}$ at $z \approx 0.2-2$. Specifically, the review will focus on massive quiescent galaxies with $M_{\text {star }} \gtrsim 10^{11} \mathrm{M}_{\odot}$ at $z_{\sim}^{<1}$, with additional coverage on quasar host halos. Empirical studies of cool/warm gas in galaxy groups and clusters of $M_{h} \gtrsim 10^{14} \mathrm{M}_{\odot}$ have been carried out for a small sample (e.g. Lopez et al., 2018; Yoon et al., 2012; Andrews et al., 2013; Stocke et al., 2014), but a detailed understanding of the gas phase in galaxy cluster and group environments relies primarily on $\mathrm{x}$-ray studies of the hot plasma. Extensive reviews on the x-ray properties of intragroup and intracluster gas can be found in Mulchaey (2000); Mathews \& Brighenti (2003); Kravtsov \& Borgani (2012).

The emphasis on quiescent galaxies in high-mass halos is motivated by the empirical finding that more than $90 \%$ of massive galaxies with $M_{\text {star }} \gtrsim 10^{11} \mathrm{M}_{\odot}$ in the local universe contain primarily evolved stellar populations with little on-going star formation (e.g. Peng et al., 2010; Tinker et al, 2013). It is therefore expected that a general understanding of the CGM properties in massive halos can be established based on observations of massive quiescent galaxies. An added bonus in studying massive quiescent galaxies is the unique opportunity to explore other feedback mechanisms for quenching star formation in massive halos, in the absence of complicated starburst driven winds.

The emphasis on quasar host halos is motivated by two factors. First, while determining quasar host mass is difficult due to uncertain host galaxy properties, the large observed clustering amplitude indicates that the mean halo mass of quasar hosts is high, $M_{h} \gtrsim 10^{12.5} \mathrm{M}_{\odot}$ (Porciani et al., 2004; White et al., 2012; Shen et al., 2013b). In addition, observations of the CGM properties in quasar host halos directly address the issues concerning the extent of AGN feedback (e.g. Shen et al., 2013a; Fumagalli et al., 2014; Rahmati et al., 2015; Faucher-Giguère et al., 2015, 2016). Most nearby elliptical galaxies are found to host a supermassive black hole at the center (Kormendy \& Richstone, 1995; Ho, 2008), and therefore high-redshift quasar hosts are likely the progenitors of these nearby massive quiescent galaxies. Studies of the CGM in quasar host halos may provide important clues for how the CGM properties are shaped while the galaxy undergoes an active quasar phase. 


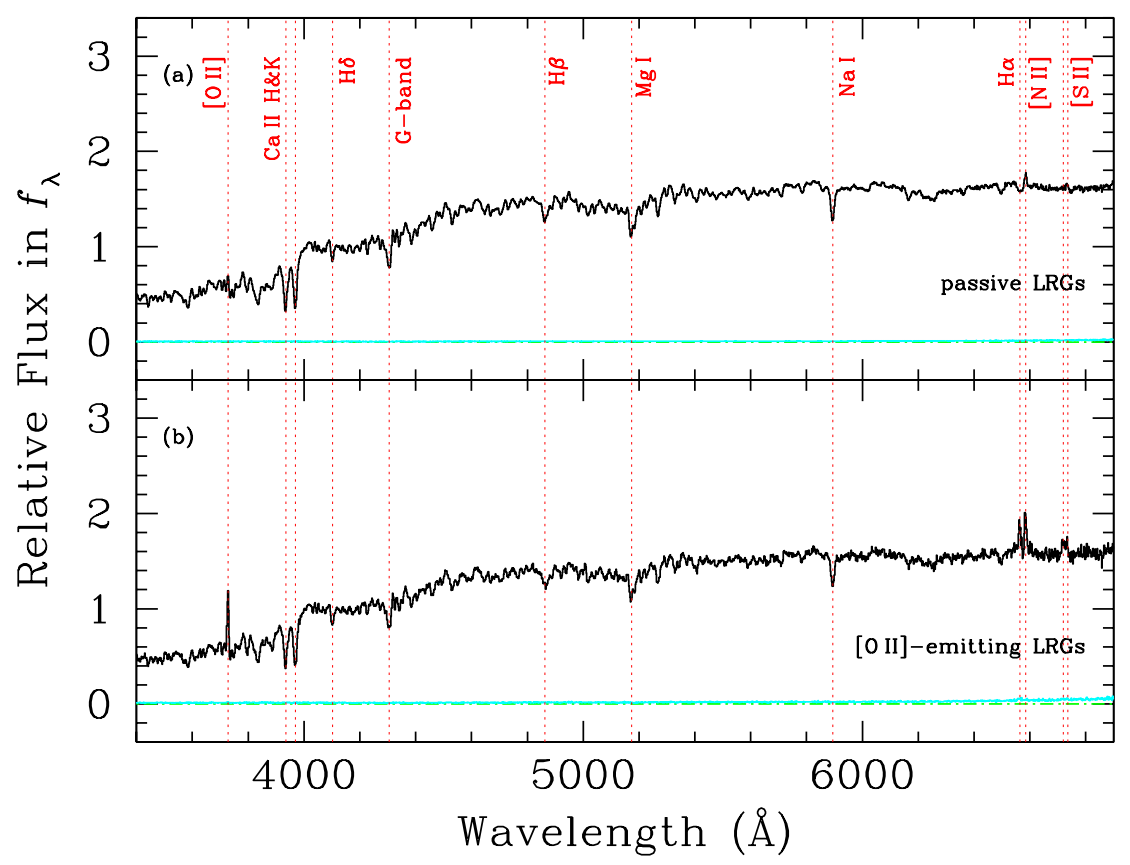

Fig. 1 The quiescent nature of $z \approx 0.5$ luminous red galaxies (LRGs) shown in stacked rest-frame optical spectra. Panel (a) shows the median stack of $\sim 1780$ passive LRGs and panel (b) shows the median stack of $\sim 280$ LRGs with [O II] emission detected (see Huang et al. (2016) for a detailed description of the sample definition and selection). Prominent absorption and emission lines are labeled in red. The corresponding 1- $\sigma$ dispersion in each stack is shown in cyan at the bottom of each panel. In both stacked spectra, the spectral properties are characterized by prominent absorption features due to $\mathrm{Ca}$ II $\mathrm{H}$ and $\mathrm{K}, \mathrm{G}-\mathrm{band}, \mathrm{Mg}$ I and $\mathrm{Na}$ I that indicate a predominantly old stellar population, as well as a relatively weak Balmer absorption series. The observed high $[\mathrm{N} \mathrm{II}] / \mathrm{H} \alpha$ emission ratios seen in both passive and [O II]-emitting LRGs, together with the low [O III]/[O II] ratios, suggest the presence of LINER in these LRGs, which could be attributed to the presence of underlying AGNs or evolved AGB stars in these galaxies.

\section{Incidence/Covering Fraction of Cool Gas in Quiescent Halos}

Luminous red galaxies (LRGs) uncovered in the SDSS exhibit little on-going star formation and display photometric and spectral properties resembling nearby elliptical galaxies (Eisenstein et al., 2001). They are characterized by a mean luminosity of $\approx 5 L_{*}$ and a mean stellar mass of $M_{\text {star }} \approx 3 \times 10^{11} \mathrm{M}_{\odot}$ at $z \approx 0.5$ (e.g. Tojeiro et al., 2011), with corresponding dark matter halo mass of $M_{h} \approx 3 \times 10^{13} \mathrm{M}_{\odot}$ (e.g. Zheng et al., 2007; Blake et al., 2008; Padmanabhan et al., 2009), and therefore offer an ideal laboratory for studying the cool gas content in massive quiescent halos.

Roughly $10 \%$ of LRGs exhibit [O II] emission features (Eisenstein et al., 2003; Roseboom et al., 2006), suggesting the possible presence of on-going star formation 
in some of these massive galaxies. However, a more detailed examination of their spectral properties reveals unusually high $[\mathrm{N} \mathrm{II]} / \mathrm{H} \alpha$ together with low [O III]/[O II] ratios, both of which indicate the presence of low-ionization nuclear emission line regions (LINER) in these galaxies (e.g. Huang et al., 2016). Figure 1 shows stacked spectra of SDSS LRGs grouped into "passive" and [O II]-emitting subsamples. In both panels of Figure 1, the stacked spectra are characterized by prominent absorption features due to $\mathrm{Ca}$ II $\mathrm{H}$ and $\mathrm{K}, \mathrm{G}-b a n d, \mathrm{Mg} I$ and $\mathrm{Na}$, along with LINER-like emission, namely strong $[\mathrm{N} \mathrm{II}] / \mathrm{H} \alpha$ ratios. The prominent absorption features indicate a predominantly old stellar population, whereas the LINER-like emission indicates the presence of additional ionizing sources in these galaxies. Although the origin of LINER-like emission is not fully understood, the observed slow decline in the spatial profile of LINER-like emission requires spatially distributed ionizing sources, rather than centrally concentrated active galactic nuclei (AGN), in these quiescent galaxies (e.g. Sarzi et al., 2006, 2010; Yan \& Blanton, 2012; Singh et al., 2013; Belfiore et al., 2016). Likely sources include photo-ionization due to hot, postasymptotic giant branch (post-AGB) stars or winds from Type Ia supernovae (e.g. Conroy et al., 2015). In the absence of on-going star formation, these LRGs also provide a unique sample for testing additional feedback mechanisms for quenching star formation in massive halos, including gravitational heating (e.g. Johansson et al., 2009) and winds from evolved stars and Type Ia supernovae (e.g. Conroy et al., 2015).

A first step toward understanding the quiescent state of LRGs is to characterize the incidence and covering fraction of cool gas in their host halos. Over the past several years, absorption-line observations of background QSOs have revealed extended cool gas at projected distances $d \approx 10-300 \mathrm{kpc}$ from LRGs at $z \sim 0.5$. Specifically, the LRG-Mg II cross-correlation function displays an amplitude that is comparable to the LRG auto-correlation on small scales ( $\underset{\sim}{\sim} 450$ comoving $\mathrm{kpc}$ ), well within the virial radii of the halos. The large clustering amplitude on small scales suggests the presence of $\mathrm{Mg}$ II gas inside these massive quiescent halos (e.g. Bouché et al., 2006; Tinker \& Chen, 2008; Gauthier et al., 2009; Lundgren et al., 2009). In addition, surveys of $\mathrm{Mg}_{\text {II }} \lambda \lambda 2796,2803$ absorption doublets in LRG halos have covered a non-negligible fraction of LRG halos with associated Mg II absorbers (e.g. Bowen \& Chelouche, 2011; Gauthier \& Chen, 2011; Gauthier et al., 2014; Zhu et al., 2014; Huang et al., 2016).

These $\mathrm{Mg}$ II absorption transitions are commonly seen at projected distances of $d_{\sim}^{<} 100 \mathrm{kpc}$ from star-forming galaxies (e.g. Lanzetta \& Bowen, 1990; Steidel et al., 1994; Nestor et al., 2007; Kacprzak et al., 2008; Chen \& Tinker, 2008; Chen et al., 2010a; Werk et al., 2013) and are understood to arise primarily in photo-ionized gas of temperature $T \sim 10^{4} \mathrm{~K}$ (e.g. Bergeron \& Stasińska, 1986; Hamann, 1997) and neutral hydrogen column density $N(\mathrm{HI}) \approx 10^{18}-10^{22} \mathrm{~cm}^{-2}$ (e.g. Churchill et al., 2000; Rao et al., 2006). The large rest wavelengths make the $\mathrm{Mg}$ II doublet a convenient probe of chemically enriched gas at $z \approx 0.3-2.3$ in optical QSO spectra. In addition, the rest-frame absorption equivalent width $W_{r}(2796)$ is found to increase proportionally with the number of individual components and the velocity spread of the components (e.g. Petitjean \& Bergeron, 1990; Churchill et al., 2000, 
2003). Measurements of $W_{r}(2796)$ therefore trace the underlying gas kinematics corresponding to $\sim 100 \mathrm{~km} \mathrm{~s}^{-1}$ per Angstrom. Several authors have proposed that strong $\mathrm{Mg}$ II absorbers of $W_{r}(2796)>1 \AA$ originate in starburst driven outflows (e.g. Zibetti et al., 2007; Ménard et al., 2011) and that the large $W_{r}(2796)$ is driven by non-gravitational motion in the outflowing media (e.g. Bouché et al., 2006). The presence of strong $\mathrm{Mg}_{\text {II }}$ absorbers near quiescent galaxies is therefore particularly surprising.

Figure 2 compares the observed mean covering fraction of $\mathrm{Mg}_{\text {II }}$ absorbing gas, $\langle\kappa\rangle_{\mathrm{Mg} \text { II }}$, in halos around typical $L_{*}$ - and sub- $L_{*}$-type, star-forming galaxies (blue triangles) with what is seen in LRG halos (green and red symbols) at $z \approx 0.3-0.5$. For a representative comparison across a broad luminosity (or mass) range, the gas covering fraction is determined within a fiducial halo gas radius, $R_{\text {gas }}$, that scales with galaxy luminosity according to $R_{\text {gas }} \approx 107\left(L_{B} / L_{B}^{*}\right)^{0.35} \mathrm{kpc}$ (Kacprzak et al., 2008; Chen et al., 2010a). In addition, $\kappa$ is estimated for halo clouds with absorption strength exceeding $W_{0}=0.3 \AA$, a detection threshold that is allowed by the quality of the absorption spectra. Previous studies have shown that typical $L_{*}$ galaxies have $R_{\text {gas }} \approx 130 \mathrm{kpc}$ and $R_{\text {gas }}$ scales with luminosity according to $R_{\text {gas }} \propto L^{0.35}$ (see Chen \& Tinker, 2008; Chen et al., 2010a). Following this scaling relation, LRGs with a mean luminosity of $\approx 3.6 L_{*}$ are expected to have $R_{\mathrm{gas}} \approx 206 \mathrm{kpc}$.

Interpreting the rest-frame $r$-band absolute magnitude as a proxy for the underlying stellar mass (e.g. Liang \& Chen, 2014), Figure 2 displays a strong mass dependence in the observed incidence and covering fraction of $\mathrm{Mg}$ II absorbing gas. Specifically, the observed $\mathrm{Mg}$ II covering fraction in LRG halos is more than four times lower than what is found in halos around lower-mass, star-forming galaxies. However, in spite of this substantially reduced cool gas content in massive quiescent halos, the distinctly non-zero $\mathrm{Mg}_{\text {II }}$ gas covering fraction around LRGs demonstrates the definitive presence of chemically-enriched cool gas around evolved galaxies. Finally, it is interesting to note that while [O II]-emitting LRGs display a slightly elevated overall cool gas content than passive LRGs, the mean gas covering fraction remains under $20 \%$ (Huang et al., 2016).

Figure 2 is based on two separate studies designed to characterize the CGM properties of $L_{*}$ /sub- $L_{*}$ galaxies using $\sim 260$ galaxies at $z \approx 0.25$ (Chen et al., 2010a) and those of massive quiescent galaxies using 38000 LRGs (Huang et al., 2016), respectively. The COS-Halos survey was designed for a systematic study of gaseous halos over a broad range of mass and star formation history, and the sample contains 28 blue sub- $L_{*}$ galaxies with a median mass of $\left\langle M_{\text {star }}\right.$ (blue $\left.)\right\rangle \approx 2 \times 10^{10} \mathrm{M}_{\odot}$ and 16 massive red galaxies with a median mass of $\left\langle M_{\text {star }}(\right.$ red $\left.)\right\rangle \approx 10^{11} \mathrm{M}_{\odot}$ (Tumlinson et al., 2013; Werk et al., 2013). While the uncertainties are large due to a smaller sample size, the COS-Halos galaxies confirm the observed mass dependence of $\mathrm{Mg}_{\mathrm{II}}$ gas covering fraction in Figure 2 (Werk et al., 2013).

A declining gas covering fraction from low-mass, star-forming galaxies to massive quiescent galaxies is also observed in H I and highly ionized species probed by the OVI $\lambda \lambda 1031,1037$ doublet transitions. Specifically, the covering fraction of moderately strong $\operatorname{Ly} \alpha$ absorbers of $W_{r}(1215)>0.1 \AA$ declines from $>90 \%$ around blue, star-forming galaxies (Tumlinson et al., 2013) to $\approx 40-50 \%$ around red pas- 


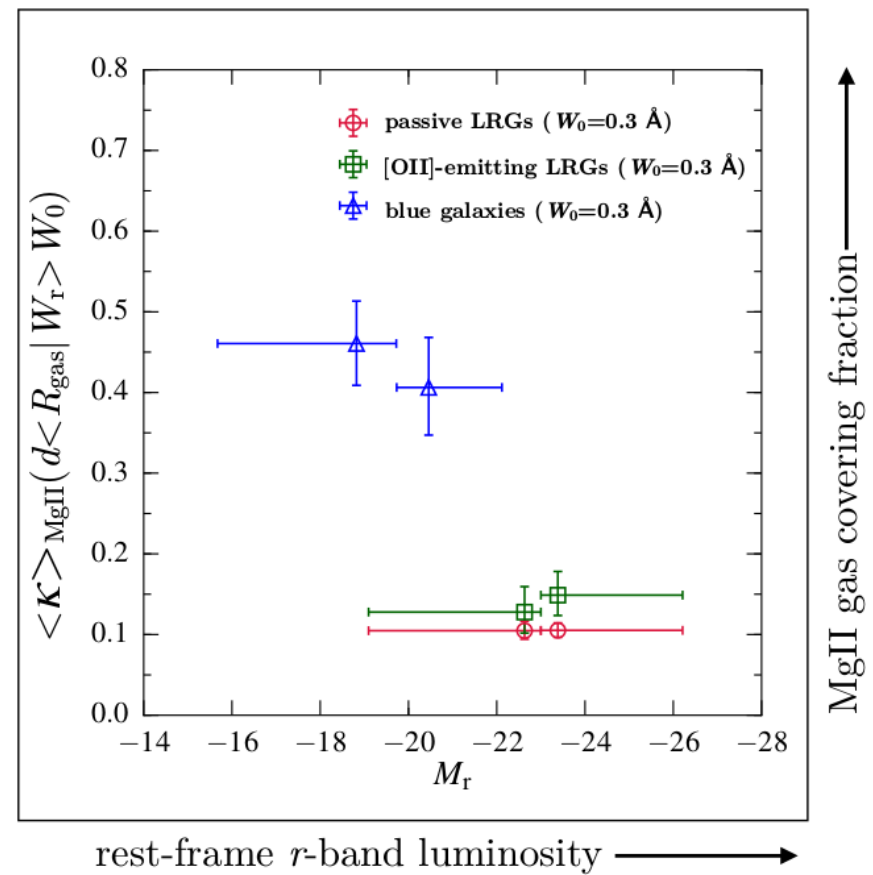

Fig. 2 Mass dependence of mean covering fraction of chemically-enriched cool gas in galaxy halos (Chen et al., 2010a; Gauthier \& Chen, 2011; Huang et al., 2016). The mean covering fraction, $\langle\kappa\rangle$, is calculated based on observations of $\mathrm{Mg}$ II absorbing gas within a fiducial halo gas radius, $R_{\text {gas }}$. Rest-frame $r$-band magnitude is adopted here as a proxy for the underlying total stellar mass (see Liang \& Chen, 2014). Star-forming galaxies at $z \approx 0.3$ are shown in blue triangles, while luminous red galaxies (LRGs) at $z \approx 0.5$ are shown in squares/circles. The covering fraction is determined for gas with absorption strength exceeding $W_{0}=0.3 \AA$, a detection threshold that is allowed by the quality of the absorption spectra. The horizontal bars represent the full range of $M_{r}$ for galaxies included in each bin, and the vertical error bars represent the $68 \%$ confidence interval. In comparison to star-forming galaxies (Chen et al., 2010a), LRGs, being old and massive, exhibit a much reduced covering fraction of chemically-enriched cool gas as probed by the $\mathrm{Mg}$ II absorption features (Gauthier \& Chen, 2011; Huang et al., 2016). However, observations also show a definitive presence of cool gas in these LRG halos, demonstrating that they are not completely devoid of star formation fuels (Huang et al., 2016). Furthermore, roughly 10\% of LRGs exhibit [O II] emission features and LINER-like spectra. These [O II]-emitting LRGs display a slightly elevated cool gas covering fraction from passive LRGs but the mean value remains significantly lower than what is seen in lower-mass, star-forming galaxies (Huang et al., 2016). Similar trends in the mean gas covering fraction have also been reported for H I Ly $\alpha$ and high-ionization species probed by the O VI $\lambda \lambda$ 1031,1037 doublet transitions. Massive quiescent galaxies also consistently display nonzero covering fractions of $\mathrm{Ly} \alpha$ and [O VI] absorbing gas (Tumlinson et al., 2011; Thom et al., 2012; Tumlinson et al., 2013; Werk et al., 2013).

sive galaxies (Thom et al., 2012), and the covering fraction of O VI absorbing gas decreases from $\approx 90 \%$ to $\approx 30 \%$ (Tumlinson et al., 2011; Werk et al., 2013). In both 
cases, massive quiescent galaxies consistently display non-zero covering fractions of Ly $\alpha$ and O VI absorbing gas.

Deep $21 \mathrm{~cm}$ and CO imaging surveys have also revealed that roughly $40 \%$ of elliptical galaxies in the nearby universe contain cool neutral gas (e.g. Oosterloo et al., 2010; Young et al., 2014). While on-going star formation is observed at low levels, $\lesssim 1 \mathrm{M}_{\odot} \mathrm{yr}^{-1}$, in many early-type galaxies of $M_{\text {star }} \approx 5 \times 10^{10} \mathrm{M}_{\odot}$, it is rarely seen in more massive systems of $M_{\text {star }} \gtrsim 10^{11} \mathrm{M}_{\odot}$ (e.g. Salim \& Rich, 2010). Combining $\mathrm{H} \mathrm{I} / \mathrm{CO}$ imaging surveys of local elliptical galaxies and absorption-line observations of distant early-type galaxies at $z \approx 0.2-0.5$ demonstrates that cool gas is indeed present in some, although not all, massive quiescent halos.

A declining cool gas fraction with increasing halo mass is expected in theoretical models that attribute the observed $\mathrm{Mg}$ II absorbers to infalling gas from either thermally unstable hot halos or the intergalactic medium (e.g. Maller \& Bullock, 2004; Kereš et al., 2009). In the presence of cool gas, it is expected that these halo clouds could provide the fuels necessary for sustaining star formation in the LRGs. The lack of on-going star formation in these massive galaxies over such an extended cosmological time period ( $\approx 2$ Gyr; Gauthier \& Chen, 2011) therefore suggests a prolonged duty cycle for the underlying heat sources.

\section{Radial Profiles of Absorbing Gas}

A key advantage of $21 \mathrm{~cm}$ and CO imaging surveys is their ability to resolve the spatial distribution of the gas around individual galaxies. The morphologies of the detected H I and CO gas span a broad range, from regular disk- or ring-like structures to irregular distributions of clumps and/or streams (e.g. Oosterloo et al., 2007; Serra et al., 2012) with roughly $1 / 4$ displaying centralized disk or ring-like structures (Serra et al., 2012). Such a wide range of morphologies suggests different origins of the gas in different galaxies, including leftover materials from previous mergers and newly accreted gas from the CGM/IGM. On the other hand, QSO absorption spectroscopy, while offering extremely sensitive probes of tenuous gas beyond the limits of $21 \mathrm{~cm} / \mathrm{CO}$ surveys, reveals gas properties along a single sightline per halo and does not provide constraints for spatial variations in individual halos. Nevertheless, considering an ensemble of close QSO and galaxy pairs over a wide range of projected separations provides a spherical average of halo absorption properties for the entire galaxy sample.

Figure 3 shows the spatial distribution of the observed $\mathrm{Mg}$ II absorption strength $\left[W_{r}(2796)\right]$ and annular average of $\mathrm{Mg}$ II gas covering fraction $\left(\langle\kappa\rangle_{\mathrm{MgII}}\right)$ as a function of projected distance $(d)$ for a sample of 13330 LRGs at $z \approx 0.5$ from Huang et al. (2016). These LRGs are selected to have a background QSO within $d \approx 500 \mathrm{kpc}$, the virial radius of a typical LRG halo, with sufficiently high signal-to-noise $(S / N)$ absorption spectra available in the SDSS archive for detecting Mg II absorbers of $W_{r}(2796) \gtrsim 0.3 \AA$. The LRGs are grouped into passive and [O II]-emitting subsamples based on the observed strength of [O II] emission lines (Figure 1). A number 
of unique features are apparent in the observed spatial distribution of chemicallyenriched cool gas in LRG halos:

- a relatively flat trend is seen in the observed $W_{r}(2796)$ versus $d$ distribution for individual absorbers uncovered over the entire projected distance range $d \underset{\sim}{<} 500$ $\mathrm{kpc}$ of both passive and [O II]-emitting LRG halos, although the scatter is large (left panel);

- a constant gas covering fraction, $\langle\kappa\rangle_{\mathrm{Mg}_{\text {II }}} \approx 15 \%$, is found at $d<120 \mathrm{kpc}$ around passive LRGs, while $\langle\kappa\rangle_{\mathrm{Mg}_{\text {II }}}$ increases from $\langle\kappa\rangle_{\mathrm{Mg}_{\text {II }}} \approx 15 \%$ at $d \approx 120 \mathrm{kpc}$ to

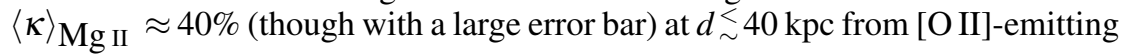
LRGs;

- the gas covering fraction declines rapidly at $d>120 \mathrm{kpc}$, reaching the expected rate of incidence due to random background absorbers at $d>300 \mathrm{kpc}$ (orange dashed line in the right panel);

- strong Mg II absorbers of $W_{r}(2796)>1 \AA$ appear to be common throughout the

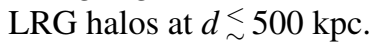

The observed flat $W_{r}(2796)$ versus $d$ trend for $\mathrm{Mg}_{\text {II }}$ absorbers and the large fraction of strong $\mathrm{Mg}_{\text {II }}$ absorbers with $W_{r}(2796)>1 \AA$ in LRG halos (see also Bowen \& Chelouche, 2011) are both in stark contrast to what is known for starforming, $L_{*}$ and sub- $L_{*}$ galaxies. Specifically, CGM observations of star-forming galaxies at both low and high redshifts have shown steadily declining absorption strength, both in hydrogen and in heavy ions, with increasing projected distance (e.g. Lanzetta \& Bowen, 1990; Bowen et al., 1995; Chen et al., 1998, 2001a; Steidel et al., 2010; Liang \& Chen, 2014). Strong Mg II absorbers with $W_{r}(2796)>1 \AA$ are only found at $d \stackrel{\sim}{\sim} 60 \mathrm{kpc}$ from isolated $L_{*}$ galaxies (e.g. Chen \& Tinker, 2008; Chen et al., 2010a). In addition, the extent of halo gas at a fixed absorption equivalent width threshold, $R_{\text {gas }}$, is found to scale with galaxy luminosity (e.g. Chen et al., 1998, 2001b; Kacprzak et al., 2008; Chen \& Tinker, 2008; Chen et al., 2010a). Therefore, the relation between $W_{r}(2796)$ versus $d / R_{\text {gas }}$ exhibits a significantly smaller scatter than the relation between $W_{r}(2796)$ and $d$. This luminosity- $R_{\text {gas }}$ scaling relation is understood as such that more luminous (and presumably more massive) halos possess more extended halo gas. For the LRGs, however, the scatter in the observed $W_{r}(2796)$ versus luminosity-normalized $d$ relation is the same as in the observed $W_{r}(2796)$ versus $d$ distribution. Figure 3 shows that, aside from a declining $\left\langle\kappa_{\mathrm{Mg}_{\text {II }}}\right\rangle$ at $d>120 \mathrm{kpc}$, the cool clouds that give rise to the observed $\mathrm{Mg}$ II absorption features do not appear to depend on either the luminosity (or equivalently mass) or projected distance of the $\mathrm{LRGs}^{1}$.

\footnotetext{
${ }^{1}$ Using stacked QSO spectra at the rest frames of all LRGs, it has been shown that the mean absorption equivalent width $\left\langle W_{0}\right\rangle$ of $\mathrm{Mg}$ II gas declines steadily with increasing projected distance in LRG halos (Zhu et al., 2014). The steady decline of $\left\langle W_{0}\right\rangle$ does not necessarily contradict the relatively flat $W_{r}(2796)$ versus $d$ distribution for individual Mg II absorbers in Figure 3, because $\left\langle W_{0}\right\rangle$ represents a weighted average of absorber strength and gas covering fraction. A direct comparison between $\left\langle W_{0}\right\rangle$ and Figure 3 requires knowledge of the frequency distribution function of Mg II absorbers in LRG halos, $f\left(W_{r}\right)$, and the underlying number density ratio between absorbing and non-absorbing LRGs.
} 

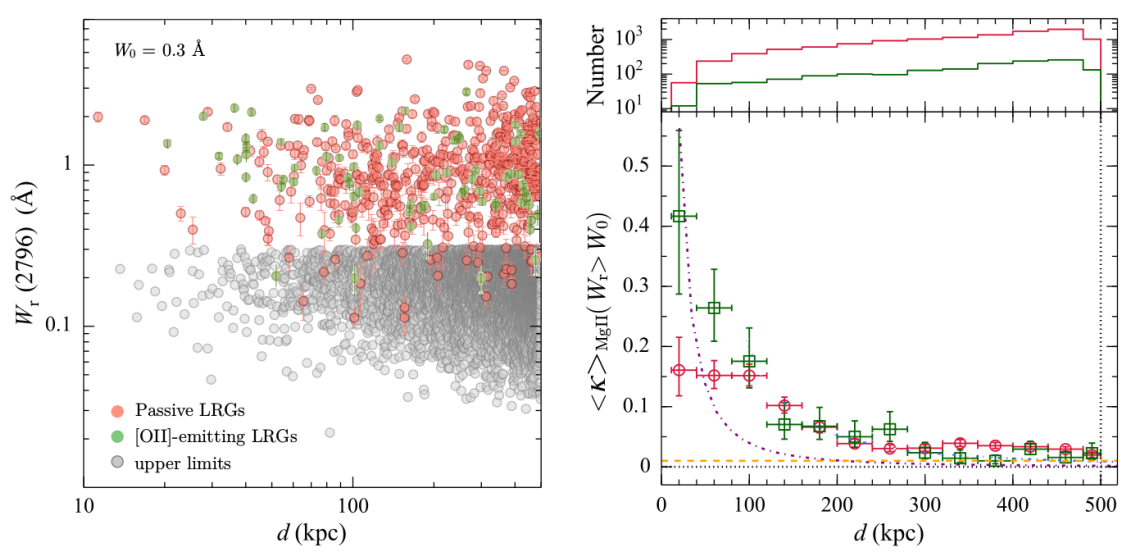

Fig. 3 Spatial distribution of the observed Mg II absorption strength $\left(W_{r}(2796)\right.$; left panel) and covering fraction $\left(\langle\kappa\rangle_{\mathrm{MgII}}\right.$; right panel) as a function of projected distance $d$ from LRGs at $z \approx 0.5$. The figures are adapted from Huang et al. (2016). The LRGs are selected to have a background QSO at $d<500 \mathrm{kpc}$ (the virial radius of a typical LRG halo) with sufficiently high signal-tonoise $(S / N)$ absorption spectra available for constraining the presence/absence of $\mathrm{Mg}$ II absorbers with absorption strength exceeding $W_{0}=0.3 \AA$. A total of 13330 LRGs in the SDSS data archive satisfy this criterion, 1575 of these exhibit [O II]-emission features and the rest are labeled as passive galaxies. For non-detections, a 2- $\sigma$ upper limit of $W_{r}(2796)$ is presented for the LRG. For a few sightlines, the QSO spectra are of sufficiently high $S / N$ for detecting $\mathrm{Mg}$ II absorption lines as weak as $W_{r}(2796) \approx 0.1 \AA$ (red and green data points with $W_{r}(2796)<0.3 \AA$ in the left panel). These are by definition included in the covering fraction calculations in the right panel, although LRGs with $W_{r}(2796)<0.3 \AA$ are considered non-absorbing galaxies at the $W_{0}=0.3-$ $\AA$ threshold. The upper-right panel displays the numbers of LRGs, passive (in red) and [O II]emitting (in green), contributing to the $\langle\kappa\rangle_{\text {MgII }}$ calculation in each $40-\mathrm{kpc}$ bin (horizontal bars in the right panel). Uncertainties in $\langle\kappa\rangle_{\text {MgII }}$ are shown in vertical error bars, which represent the $68 \%$ confidence intervals. Possible contributions due to random background absorbers and gasrich satellite galaxies are also shown in the orange dashed line and the purple dash-dotted curve, respectively.

The lack of dependence of the observed Mg II absorbing clouds on the LRG properties appears to rule out the possibility of these absorbers being connected to outgoing materials from the LRGs. However, the observed flat mean covering fraction

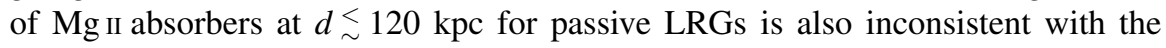
expectation for accreted materials from the IGM, which are expected to show an increasing covering fraction with decreasing projected distance (e.g. Faucher-Giguère \& Kereš, 2011; Fumagalli et al., 2011; Shen et al., 2013a; Ford et al., 2014). Such a discrepancy indicates that IGM accretion alone cannot explain the spatial distribution of chemically-enriched cool gas in these massive quiescent halos, at least not in the inner $120 \mathrm{kpc}$.

Contributions from the interstellar and circumgalactic gas of satellite galaxies to the observed Mg II absorbers in LRG halos have also been considered (e.g. Gauthier et al., 2010; Huang et al., 2016). In particular, observations have shown that LRGs 
reside in overdense environments with neighboring satellites (e.g. Tal et al., 2012). Roughly $20 \%$ of satellite galaxies are blue and presumably gas-rich (e.g. Hansen et al., 2009; Prescott et al., 2011). Under the assumption that these blue satellites can retain their gaseous halos, the expected contributions to the observed covering fraction of $\mathrm{Mg}_{\text {II }}$ gas can be estimated based on the known luminosity scaling relation (e.g. Chen \& Tinker, 2008). The expectation is shown in the purple curve in the right panel of Figure 3. It is clear that blue satellites alone cannot account for the observed $15 \%$ covering fraction of $\mathrm{Mg}$ II absorbing gas at $d \sim 100 \mathrm{kpc}$ in LRG halos.

A promising explanation for the observed $\mathrm{Mg}$ II absorbers in LRG halos are cool clumps condensing out of thermally unstable hot halos (e.g. Mo \& MiraldaEscudé, 1996; Maller \& Bullock, 2004; Voit et al., 2015). In particular, hot halos are a common feature among the LRG population, because efforts in search of a Sunyaev-Zel'dovich signal (Sunyaev \& Zeldovich, 1970) around high-mass LRGs $\left(M_{h}>8 \times 10^{13} \mathrm{M}_{\odot}\right)$ have yielded detections in all mass bins studied (Hand et al., 2011). In addition, $\mathrm{Mg}_{\text {II }}$ absorbers are frequently resolved into multiple components with the number of components being proportional to both the rest-frame absorption equivalent width $W_{r}(2796)$ (e.g. Petitjean \& Bergeron, 1990; Churchill et al., 2003) and the minimum line-of-sight velocity spread (e.g. Churchill et al., 2000). The Mg II absorption equivalent width is therefore driven by the line-of-sight cloud motion rather than by the underlying total gas column density. Recall also that the $\mathrm{Mg}$ II absorbers found in the vicinities of passive and [O II]-emitting LRGs display a constant $W_{r}(2796)$ across the entire projected distance range shown in Figure 3 with a mean and dispersion of $\left\langle\log W_{r}(2796) / \AA\right\rangle=-0.050 \pm 0.25$.

Interpreting the scatter as due to Poisson noise in the number of individual clumps intercepted along a line of sight leads to an estimate of the mean number of clumps per galactic halo per sightline, $n^{\text {clump }}$ (e.g. Lanzetta \& Bowen, 1990; Chen et al., 2010a). In this simple toy model, each absorber is characterized as $W_{r}=n^{\text {clump }} \times \omega_{0}$, where $\omega_{0}$ is the mean absorption equivalent width per component. Following Poisson counting statistics, the intrinsic scatter $\delta\left(\log W_{r}\right) \equiv$ $\delta\left(W_{r}\right) /\left(W_{r} \ln 10\right)$ is related to the mean number of clumps $\left\langle n^{\text {clump }}\right\rangle$ according to

$$
\delta\left(\log W_{r}\right)=\frac{1}{\ln 10} \frac{\sqrt{\left\langle n^{\text {clump }}\right\rangle+1}}{\left\langle n^{\text {clump }}\right\rangle},
$$

which leads to $\left\langle n^{\text {clump }}\right\rangle \sim 3.8$ for $\left\langle W_{r}(2796)\right\rangle \approx 1 \AA$ in the LRG halos with a corresponding mean absorption equivalent width per component of $\omega_{0}=0.24 \AA$. The inferred $\left\langle n^{\text {clump }}\right\rangle$ per sightline for LRG halos is more than four times smaller than what was inferred for lower-mass, star-forming galaxies at $d \approx 40 \mathrm{kpc}$ (Chen et al., 2010a). With a halo size twice as big as $L_{*}$ galaxies, the significantly lower $\left\langle n^{\text {clump }}\right\rangle$ therefore suggests that the volume filling factor of $\mathrm{Mg}$ II absorbing gas is $\sim 10$ times lower in LRG halos than in $L_{*}$ halos. 


\section{Kinematics}

While obtaining spatially-resolved velocity maps is challenging for both stellar and gaseous components of individual high-redshift galaxies, important insights into the physical nature of cool halo clouds can be gained from comparing the distribution of relative velocities between absorbers and their associated galaxies. One important observable of cool halo gas is the line-of-sight velocity dispersion within the host halo. Using a sample of galaxy and absorber pairs, it is possible to constrain the ensemble average of the velocity distribution of absorbing gas relative to their host galaxies.

The distribution of relative velocity between $\mathrm{Mg}_{\text {II }}$ absorbers and their host galaxies is shown in Figure 4 for both passive and [O II]-emitting LRGs. For the two LRG subsamples, the relative motion of $\mathrm{Mg}$ II absorbing gas is well centered around the systemic redshifts of the galaxies. While the velocity distribution around [O II]-emitting galaxies is well characterized by a single Gaussian of dispersion $\sigma_{v}=167 \mathrm{~km} \mathrm{~s}^{-1}$, a double Gaussian profile is required to characterize the relative motion of $\mathrm{Mg}$ II gas around passive LRGs with a narrow component of $\sigma_{v}^{n}=163$ $\mathrm{km} \mathrm{s}^{-1}$ for the majority of the sample and a broad component of $\sigma_{v}^{b}=415 \mathrm{~km} \mathrm{~s}^{-1}$. Roughly $12 \%$ of passive LRGs are found in the broad component with velocities exceeding $500 \mathrm{~km} \mathrm{~s}^{-1}$. These properties apply to both inner $(d<200 \mathrm{kpc})$ and outer (200<d<500 kpc) halos (see Huang et al., 2016, for a detailed discussion).

The implications of Figure 4 are two-fold. First of all, the similarity in the relative velocity distribution of $\mathrm{Mg}$ II absorbing gas around passive and [O II]-emitting LRGs reaffirms the findings from the previous section (Section 3) that the observed Mg II absorbers are unlikely to originate in outgoing materials from the LRGs. Secondly, the mean halo mass of LRGs is $M_{h} \approx 3 \times 10^{13} M_{\odot}$ (e.g. Mandelbaum et al., 2008; Gauthier et al., 2009). The expected line-of-sight velocity dispersion for virialized gas in these massive halos is $\sigma_{h} \approx 265 \mathrm{~km} \mathrm{~s}^{-1}$. The observed velocity dispersion of $\mathrm{Mg}$ II absorbing gas in $\approx 90 \%$ of the LRG sample (the narrow component in Figure 4) is merely $60 \%$ of what is expected from virial motion (see also Zhu et al., 2014), namely

$$
\sigma_{v} \approx 0.6 \sigma_{h}
$$

Such suppression in gas motion not only indicates that the gas is gravitationally bound in the LRG halo but also that the kinetic energy of the gas is being dissipated. Similar suppression in the line-of-sight velocity dispersion is also found for Ly $\alpha$ and O VI absorbing gas around COS-Halos red galaxies with $M_{\text {star }}>10^{10.7} \mathrm{M}_{\odot}$ (Tumlinson et al., 2011, 2013).

In the presence of a hot halo (e.g. Hand et al., 2011), cool clumps should experience a ram-pressure drag force and slow down. For ram-pressure to drive decelerations in the clump motion, these clumps cannot be too massive. The observed suppression in the velocity dispersion of the absorbing gas therefore places a mass limit on these clumps. Following the formalism of Maller \& Bullock (2004), a maximum clump mass can be estimated for the LRG halos according to $m_{\mathrm{cl}} \approx 5.1 \times 10^{4} M_{\odot} T_{6}^{-3 / 8}\left(\Lambda_{z} t_{8}\right)^{1 / 2}$, where $T_{6}$ is the halo gas temperature in units 

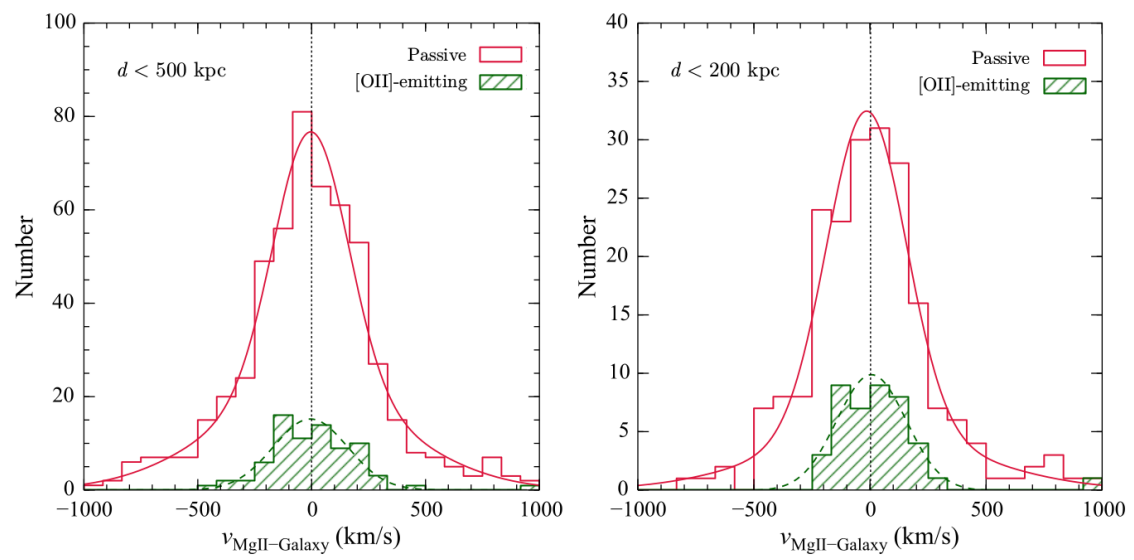

Fig. 4 Velocity distributions of Mg II -absorbing clumps relative to the systemic redshifts of LRGs based on an ensemble of Mg II absorber and LRG pairs (adapted from Huang et al., 2016). The relative velocity distribution of $\mathrm{Mg}$ II -absorbing gas relative to passive LRGs is shown in the red open histogram, while the velocity distribution of $\mathrm{Mg}$ II absorbing gas relative to [O II]-emitting LRGs is shown in the green hatched histogram. Including all Mg II absorbers at $d<500 \mathrm{kpc}$ of an LRG (left panel), the velocity distribution of the gas is well characterized by a single Gaussian distribution centered at $\left\langle v_{\mathrm{MgII}-\text { Galaxy }}\right\rangle=-5 \mathrm{~km} \mathrm{~s}^{-1}$ with a dispersion of $\sigma_{v}=167 \mathrm{~km} \mathrm{~s}^{-1}$ (green, dashed curve). At the same time, a double Gaussian profile is required to better characterize the velocity distribution of $\mathrm{Mg}$ II absorbing gas around passive LRGs (red solid curve) with a narrow component centered at $\left\langle v_{\mathrm{MgII}-\text { Galaxy }}\right\rangle=-3 \mathrm{~km} \mathrm{~s}^{-1}$ and $\sigma_{v}^{n}=163 \mathrm{~km} \mathrm{~s}^{-1}$ and a broad component centered at $\left\langle v_{\mathrm{MgII}-\text { Galaxy }}\right\rangle=-17 \mathrm{~km} \mathrm{~s}^{-1}$ and $\sigma_{v}^{b}=415 \mathrm{~km} \mathrm{~s}^{-1}$. Roughly $12 \%$ of passive LRGs have a Mg II absorber found at velocities exceeding $500 \mathrm{~km} \mathrm{~s}^{-1}$ from the systemic redshifts of the galaxies. Consistent velocity distributions are found for $\mathrm{Mg}$ II absorbers detected at $d<200 \mathrm{kpc}$ from passive and [O II]-emitting galaxies (right panel), showing little variation in the overall gas motion between inner and outer halos.

of $10^{6} \mathrm{~K}, \Lambda_{z}$ is the cooling parameter that varies with the gas metallicity $\mathrm{Zg}_{\mathrm{g}}$ and $t_{8}=t_{f}\left(c_{h}\right) / 8 \mathrm{Gyr}$ is the halo formation time that depends on the halo concentration $c_{h}$. For a halo temperature of $T \sim 6 \times 10^{6} \mathrm{~K}$ for the LRGs and $t_{8} \sim 8.9 \mathrm{Gyr}$ using $c_{h} \sim 10$ from the halo mass-concentration relation (e.g. Mandelbaum et al., 2008), the estimated mass of individual $\mathrm{Mg}$ II absorbing clumps is $m_{\mathrm{cl}} \approx 5 \times 10^{4} M_{\odot}$ for solar metallicity and lower for lower-metallicity (see Huang et al., 2016, for more details).

Beyond an ensemble average over a large sample of LRG-Mg II pairs, comparisons of gas and satellite kinematics in individual halos are possible when deep galaxy survey data are available (e.g. Steidel et al., 1997; Whiting et al., 2006; Nestor et al., 2007; Chen \& Mulchaey, 2009). Previous studies that combined absorber and galaxy data have revealed a number of metal-line absorbers associated with overdensities of galaxies over a wide range of mass scales (e.g. Mulchaey \& Chen, 2009; Kacprzak et al., 2010; Nestor et al., 2011; Gauthier, 2013; Johnson et al., 2013). Comparing the observed line-of-sight velocity dispersion of the absorbing gas with the velocity distribution of galaxy group members offers additional clues 
for the origin of chemically-enriched gas in overdense environments. In particular, imaging and spectroscopic surveys of galaxies in the vicinities of ultra-strong $\mathrm{Mg}$ II absorbers, $W_{r}(2796) \approx 3 \AA$, have revealed multiple galaxies at small projected distances and velocity separations from these absorbers (e.g. Nestor et al., 2007, 2011; Gauthier, 2013). With the expected velocity spread of $\sim 100 \mathrm{~km} \mathrm{~s}^{-1}$ per Angstrom for $\mathrm{Mg}$ II absorbers, the large $W_{r}$ (2796) implies a line-of-sight velocity spread of the components $\Delta v>300 \mathrm{~km} \mathrm{~s}^{-1}$. Attributing the observed gas motion to the underlying gravitational potential of the host dark matter halo would lead to a halo mass exceeding $M_{h} \approx 3 \times 10^{12} \mathrm{M}_{\odot}$.

Figure 5 showcases an example of an ultra-strong $\mathrm{Mg}$ II absorber of $W_{r}(2796)=$ $4.2 \AA$ identified at $z=0.5624$ along the sightline toward a background QSO at $z_{\mathrm{QSO}}=1.30$ (Gauthier, 2013). Four galaxies are spectroscopically identified at close projected distances and velocity separations from the absorber, including a 3.5- $L_{*}$ LRG at $d=246 \mathrm{kpc}$ and $\Delta v=-385 \mathrm{~km} \mathrm{~s}^{-1}$, a $1.8-L_{*}$ passive galaxy $(A)$ at $d=55$ $\mathrm{kpc}$ and $\Delta v \approx 0 \mathrm{~km} \mathrm{~s}^{-1}$, a $0.3-L_{*}$ galaxy $(B)$ at $d=38 \mathrm{kpc}$ and $\Delta v \approx-100 \mathrm{~km} \mathrm{~s}^{-1}$, and a $0.5-L_{*}$ galaxy $(G)$ at $d=209 \mathrm{kpc}$ and $\Delta v \approx 0 \mathrm{~km} \mathrm{~s}^{-1}$. Only galaxies $B$ and $G$ exhibit traces of on-going star formation with an inferred star formation rate of $\approx 3 \mathrm{M}_{\odot} \mathrm{yr}^{-1}$. The total stellar masses of the galaxies range from $M_{\text {star }} \approx 4 \times 10^{10} \mathrm{M}_{\odot}$ for galaxy $A$ and the LRG to $M_{\text {star }} \approx 3 \times 10^{9} \mathrm{M}_{\odot}$ for galaxies $B$ and $G$. The close proximity and the observed massive stellar content qualify these galaxies as parts of a galaxy group with a light-weighted center at $d_{\text {group }} \approx 156 \mathrm{kpc}$ and $\Delta v_{\text {group }}=-200$ $\mathrm{km} \mathrm{s}^{-1}$. The total dark matter halo mass of the group estimated based on the stellar mass of the LRG is $M_{h} \approx 10^{13} \mathrm{M}_{\odot}$ (e.g. Behroozi et al., 2010).

The star formation rate intensity observed in galaxies $B$ and $G$ is small (Gauthier, 2013), which indicates that super galactic winds are unlikely to form and drive outflows in these galaxies (e.g. Heckman, 2002; Heckman et al., 2015). On the other hand, the large line-of-sight velocity spread of the absorbing gas, from $\Delta v \approx-250$ to $\Delta v \approx 230 \mathrm{~km} \mathrm{~s}^{-1}$ (lower-right panel), is comparable to the projected velocity dispersion expected from virial motion, $\sigma_{\mathrm{vir}} \approx 190 \mathrm{~km} \mathrm{~s}^{-1}$, in a halo of $M_{h} \approx 10^{13} \mathrm{M}_{\odot}$ at $z=0.5$. This agreement suggests a physical connection between group dynamics and ultra-strong $\mathrm{Mg}$ II absorbers. A comparable velocity dispersion between absorbing gas and group galaxies has also been found for a $\mathrm{Mg}$ II absorber of $W_{r}(2796)=1.8 \AA$ at $z=0.3$, for which a group of five sub- $L_{*}$ galaxies are found at $d<250 \mathrm{kpc}$ and $|\Delta v|<250 \mathrm{~km} \mathrm{~s}^{-1}$ (Kacprzak et al., 2010). The dynamical mass of the host dark matter halo, based on the velocity dispersion of the group members $\sigma_{v}=115 \mathrm{~km} \mathrm{~s}^{-1}$, is $M_{h} \approx 3 \times 10^{12} \mathrm{M}_{\odot}$ for the galaxy group at $z=0.3$. In both cases, where a strong $\mathrm{Mg}_{\text {II }}$ is found in a galaxy group, dissipation is not observed in the relative motion between the absorbing gas and associated galaxies. The large velocity dispersion, similar to the broad velocity component seen in Figure 4, can be understood if the ram-pressure drag force is subdominant in comparison to the gravitational force on the gas clumps. Stripped gas due to tidal interactions between neighboring galaxies is a likely explanation for the dynamically complex Mg II absorbers found in group or cluster environments (e.g. Chynoweth et al., 2008; Marasco et al., 2016), which also results in H I depleted galaxies in these 

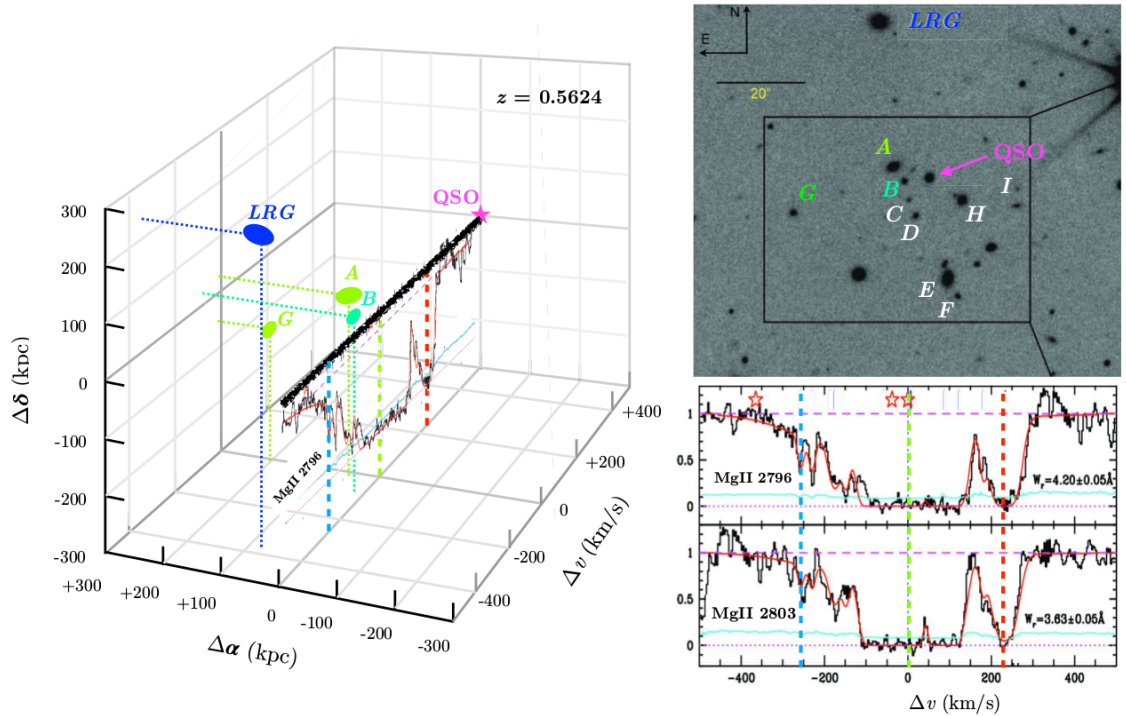

Fig. 5 Comparison of galaxy and $\mathrm{Mg}$ II absorption gas kinematics observed in a group of galaxies at $z=0.5624$ (Gauthier, 2013). The imaging panel and absorption-line profiles on the right are adapted from Gauthier (2013). An ultra-strong Mg II absorber of $W_{r}(2796)=4.2 \AA$ (lower-right panel) is identified at $z=0.5624$ along the sightline toward a background QSO at $z$ QSO $=1.30$. Follow-up spectroscopy of galaxies in this field (upper-right panel) revealed an LRG at $d=246$ $\mathrm{kpc}$ and $\Delta v=-385 \mathrm{kms}^{-1}$, along with three additional galaxies, $A, B$, and $G$ at closer distances and relative velocities from the $\mathrm{Mg}$ II absorber. Galaxies $C-F, H$, and $I$ are found to be at lower redshifts from the galaxy group at $z=0.56$. The left panel displays the spatial and relative velocity distribution of the LRG and three neighboring galaxies relative to the Mg II absorber. The luminosities of the LRG and galaxies $A, B$, and $G$ are $3.5,1.8,0.3$, and $0.5 L_{*}$, respectively. The galaxies are color-coded according to the relative velocities from the Mg II absorber at $\Delta v=0$ $\mathrm{km} \mathrm{s}^{-1}$. Galaxies $A$ and $G$, marked in pale green, occur at $\Delta v \approx 0 \mathrm{~km} \mathrm{~s}^{-1}$, while the LRG, marked in blue, displays the largest blue-shifted relative velocity with $\Delta v=-385 \mathrm{~km} \mathrm{~s}^{-1}$. The most blueshifted and redshifted Mg II components at $\Delta v \approx-250$ and $230 \mathrm{~km} \mathrm{~s}^{-1}$ are also marked in pale blue and red, respectively, to guide the visual comparison.

massive cluster and group halos (e.g. Verdes-Montenegro et al., 2001; Chung et al., 2009).

Galaxy surveys have been carried out for two more ultra-strong $\mathrm{Mg}$ II absorbers at $z \approx 0.7$. Both are found in a group environment with $M_{h}=10^{12-13} \mathrm{M}_{\odot}$ (Nestor et al., 2011). However, high-resolution spectra are not available for these two absorbers and consequently comparisons of gas and galaxy kinematics are not possible. 


\section{Chemical Enrichment}

Figure 3 clearly indicates that despite being in a quiescent state, halo gas around massive LRGs has been enriched with heavy elements out to the virial radius. The chemical enrichment history in these massive halos, which can be broadly characterized by (i) gas metallicity and (ii) relative abundance pattern, offers independent clues for the origin of the observed cool halo gas. Specifically, gas metallicity quantifies the overall heavy element production level, and nearby galaxies are observed to follow a mass-metallicity relation with more massive galaxies displaying on average higher metallicities both in stars (Gallazzi et al., 2005; Kirby et al., 2013) and in the ISM (Tremonti et al., 2004; Lee et al., 2006). Therefore, a natural expectation is that gas ejected from massive galaxies should be highly metal-enriched, while gas ejected from low-mass satellites should exhibit a lower metallicity. Gas accreted from the IGM should contain still lower metallicities (e.g. Lehner et al., 2013; Kacprzak et al., 2014). However, uncertainties arise as a result of poorly understood processes involving mixing and transport of heavy elements (e.g. Tumlinson, 2006). If mixing is effective as metal-enriched materials propagate into the low-density, and presumably lower-metallicity, halo gas, then the overall metallicity is expected to be reduced. If mixing is ineffective, then large variations in chemical abundances are expected in single objects (e.g. Scale \& Elmegreen, 2004). Consequently, metallicity provides only a weak indicator for possible origins of chemically-enriched cool gas in LRG halos.

On the other hand, the relative abundance pattern presents a fossil record for the primary source of heavy element production. Specifically, $\alpha$-elements (such as $\mathrm{O}$, $\mathrm{Mg}, \mathrm{Si}$, and $\mathrm{S}$ ) are primarily produced in massive stars and core-collapse supernovae $(\mathrm{SNe})$, while a significant fraction of heavier elements such as $\mathrm{Fe}$ and $\mathrm{Ni}$ are produced in Type Ia SNe over longer timescales (e.g. Tsujimoto et al., 1995; Nomoto et al., 2006). The observed $[\alpha / \mathrm{Fe}]$ relative abundance ratio therefore constrains the relative contributions of core-collapse $\mathrm{SNe}$ and $\mathrm{SNe}$ Ia to the chemical enrichment history (Tinsley, 1979; Gilmore \& Wyse, 1991; Tolstoy et al., 2003). Both distant galaxies (Wolfe et al., 2005) and nearby evolved stars (Matteucci, 2014) display an $\alpha$-element enhanced abundance pattern, indicating a chemical enrichment process driven by core-collapse $\mathrm{SNe}$ in the early universe. At the same time, the ISM of nearby elliptical galaxies (e.g. Mathews \& Brighenti, 2003; Humphrey \& Buote, 2006) and intracluster medium (e.g. de Plaa et al., 2007) display super-solar Fe/ $\alpha$ abundance ratios that reveal increased contributions from SNe Ia in the circumgalactic gas of massive, evolved galaxies. Relative abundance measurements between $\mathrm{Fe}$ and $\alpha$-elements therefore provide a powerful constraint for the origin of chemicallyenriched gas around galaxies.

At $z \gtrsim 0.3$, a series of Fe II absorption transitions are observable on the ground, which enable an accurate measurement of Fe II column density $N(\mathrm{Fe}$ II $)$ on a component by component basis (e.g. Rigby et al., 2002; Narayanan et al., 2008; Zahedy et al., 2016a). Similarly, the Mg II doublet transitions enable accurate Mg II column density measurements $N(\mathrm{Mg}$ II $)$ for all but a few saturated components. In principle, the total elemental abundances can be determined from the observed $N\left(\mathrm{Fe}_{\mathrm{II}}\right)$ and 

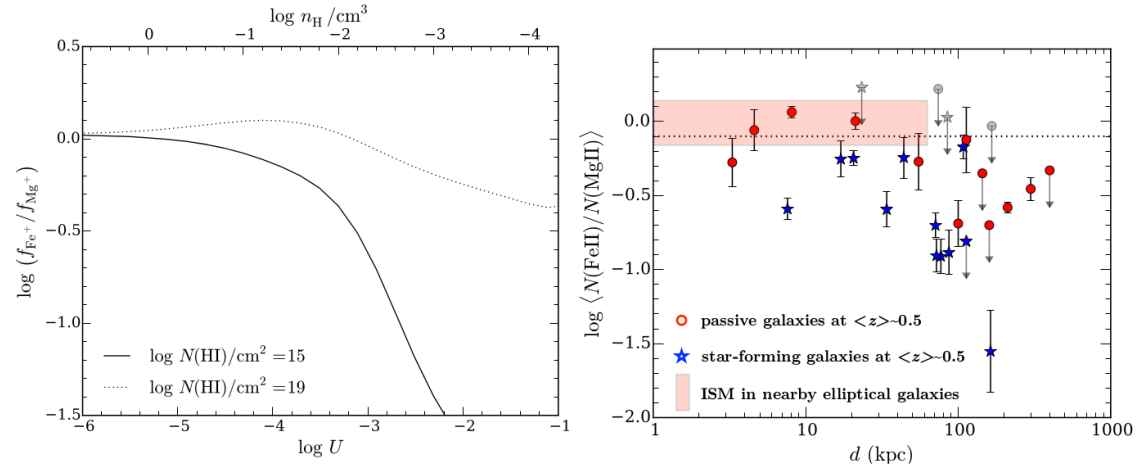

Fig. 6 Enhanced Fe abundances in halos around massive quiescent galaxies in comparison to those around star-forming galaxies (Zahedy et al., 2016a,b). Left: Expected ionization fractions of $\mathrm{Mg}^{+}$ and $\mathrm{Fe}^{+}$ions from Cloudy photo-ionization calculations (Ferland et al., 2013) for photo-ionized gas of temperature $T=10^{4} \mathrm{~K}$ and 0.1 solar metallicity in optically-thin (with neutral hydrogen column density $N(\mathrm{HI})=10^{15} \mathrm{~cm}^{-2}$ ) and optically-thick (with $N(\mathrm{HI})=10^{19} \mathrm{~cm}^{-2}$ ) regimes (Zahedy et al., 2016a). Changing metallicity does not alter the results. An updated version of the Haardt \& Madau (2001) ionizing radiation field at $z=0.5$ is adopted for computing the ionization parameter $U$ at different gas density $n_{\mathrm{H}}$ displayed at the top of the panel. These calculations demonstrate that, independent of gas metallicity, the observed $N(\mathrm{Fe}$ II $) / N(\mathrm{Mg}$ II $)$ in the right panel represents a lower limit to the underlying ( $\mathrm{Fe} / \mathrm{Mg}$ ) relative abundances. Right: Observed mean Fe II to Mg II column density ratio versus projected distance based on absorption spectroscopy of halo gas around quiescent (red circles) and star-forming (blue stars) galaxies at $\langle z\rangle \approx 0.5$. For comparison, ISM measurements based on x-ray observations of 19 nearby elliptical galaxies (Humphrey \& Buote, 2006) are also included in the left panel, and the dotted line shows the solar value. The fractional contribution of Type Ia supernovae to the chemical enrichment history in the inner $60 \mathrm{kpc}$ of massive quiescent halos is found to be similar to what is observed in the solar neighborhood.

$N \mathrm{Mg}$ II ), if the ionization fractions of these ions are known. Gas metallicity can also be derived if the total hydrogen content can be inferred from measurements of the neutral hydrogen column density, $N(\mathrm{HI})$. In practice, observations of $N(\mathrm{HI})$ at low redshifts require UV spectroscopy carried out in space, and have only been done for a relatively small sample of galaxies (e.g. Chen \& Prochaska, 2000; Werk et al., 2014). Furthermore, uncertainties in the derived ionization fractions of individual ions can be large and difficult to estimate under simple ionization models.

Observations of the relative abundance ratio between $\mathrm{Mg}^{+}$and $\mathrm{Fe}^{+}$ions are particularly useful, because $\mathrm{Mg}^{+}$and $\mathrm{Fe}^{+}$share similar ionization potentials (15 $\mathrm{eV}$ and $16.2 \mathrm{eV}$, respectively) and are the dominant ionization states of the respective elements in both neutral and cool photo-ionized gas. While the elemental abundances of iron and magnesium may be uncertain, the relative abundance ratio, $(\mathrm{Fe} / \mathrm{Mg})$ can be inferred with high confidence from the observed $N(\mathrm{Fe} \mathrm{II}) / N(\mathrm{Mg}$ II following $\log (\mathrm{Fe} / \mathrm{Mg})=\log N(\mathrm{Fe}$ II $) / N(\mathrm{Mg}$ II $)-\log \left(f_{\mathrm{Fe}^{+}} / f_{\mathrm{Mg}^{+}}\right)$, where $f_{\mathrm{Fe}^{+}}$is the fraction of $\mathrm{Fe}$ in the singly ionized state and $f_{\mathrm{Mg}^{+}}$is the fraction of $\mathrm{Mg}$ in the singly ionized state. The ratio of ionization fractions, $f_{\mathrm{Fe}^{+}} / f_{\mathrm{Mg}^{+}}$, estimated based on a suite of photo-ionization calculations is shown in the left panel of Figure 6 . 
The photo-ionization models are computed using the Cloudy software (Ferland et al., 2013). These models assume a photo-ionized gas of temperature $T=10^{4} \mathrm{~K}$ and 0.1 solar metallicity in optically-thin (with neutral hydrogen column density $N(\mathrm{HI})=10^{15} \mathrm{~cm}^{-2}$ ) and optically-thick (with $N(\mathrm{HI})=10^{19} \mathrm{~cm}^{-2}$ ) regimes (Zahedy et al., 2016a). An updated version of the Haardt \& Madau (2001) ionizing radiation field at $z=0.5$ is adopted for computing the ionization parameter $U$ (defined as the number of ionizing photons per atom) at different gas density $n_{\mathrm{H}}$ displayed at the top of the panel. The ionization fraction of $\mathrm{Fe}^{+}$remains roughly equal to that of $\mathrm{Mg}^{+}$ in the optically-thick regime and lower in optically-thin gas for the full range of $U$ explored. Consequently, $\log \left(f_{\mathrm{Fe}^{+}} / f_{\mathrm{Mg}^{+}}\right) \lesssim 0$ and the observed $N(\mathrm{Fe}$ II $) / N(\mathrm{Mg}$ II $)$ marks a lower limit to the underlying Fe to $\alpha$-element abundance ratio:

$$
\log \left(\frac{\mathrm{Fe}}{\mathrm{Mg}}\right)>\log \frac{N(\mathrm{Fe} I \mathrm{II})}{N(\mathrm{MgII})} .
$$

Experimenting with different gas metallicity does not change the predicted ionization fraction, and accounting for differential dust depletion of iron and magnesium would further increase the inferred $[\mathrm{Fe} / \mathrm{Mg}]$ (Savage \& Sembach, 1996). This exercise demonstrates that useful empirical constraints for the relative $\mathrm{Fe}$ and $\mathrm{Mg}$ abundances can be obtained even in the absence of accurate measurements of $N(\mathrm{HI})$ and gas metallicity.

A recent study utilizing multiply-lensed QSOs for probing gas at small pro-

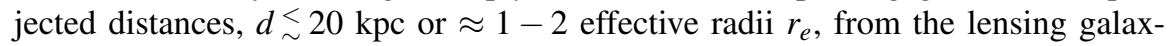
ies has uncovered important new clues for the origin of chemically-enriched cool gas in massive halos (Zahedy et al., 2016a). These lensing galaxies at $z=0.4-0.7$ share similar properties concerning both the quiescent state and halo mass scales $\left(M_{h} \gtrsim 10^{13} \mathrm{M}_{\odot}\right)$, but display distinct absorption-line profiles between different lensing galaxies and between different sightlines of individual lenses. The apparent large scatter in the observed absorption profiles is consistent with the large scatter displayed in Figure 3 and discussed in Section 3. Most interestingly, all Mg II absorbers detected near these lensing galaxies are strong and resolved into $8-15$ individual components over a line-of-sight velocity range of $\Delta v \approx 300-600 \mathrm{~km} \mathrm{~s}^{-1}$. The $\mathrm{Mg}$ II absorption is accompanied with even stronger Fe II absorption with matching kinematic profiles. Comparing the relative absorption strengths between individual components also yields uniformly large $N(\mathrm{Fe}$ II $) / N(\mathrm{Mg}$ II $)$ ratios over the full range of velocity spread, $\Delta v$, with a median of $\langle\log N(\mathrm{Fe}$ II $) / N(\mathrm{Mg}$ II $)\rangle \approx 0$ and a scatter of $<0.1$ dex.

Following Equation (3), the observed $\langle\log N(\mathrm{Fe}$ II $) / N(\mathrm{Mg}$ II $)\rangle \approx 0$ naturally leads to a super solar $\mathrm{Fe} / \mathrm{Mg}$ abundance ratio near these massive lensing galaxies. This is in stark contrast to an $\alpha$-element enhanced chemical composition found in young, star-forming galaxies (Dessauges-Zavadsky et al., 2004; Crighton et al., 2013; Fox et al., 2014, e.g.) and see Zahedy et al. (2016a) for a detailed comparison, and clearly indicates different origins between gas associated with star-forming galaxies and with massive quiescent galaxies. 
Previous studies have shown that the spatial distribution of SNe Ia in nearby early-type galaxies follows the stellar light out to $r \sim 4 r_{e}$ (e.g. Förster \& Schawinski, 2008) and that the ISM of these massive quiescent galaxies exhibits an ironenhanced abundance pattern (e.g. Mathews \& Brighenti, 2003; Humphrey \& Buote, 2006). Therefore, it seems likely that the observed Fe-rich gas at $d \approx r_{e}$ from the lenses originates in the ISM of these massive galaxies, where SNe Ia play a dominant role in driving the observed large velocity width and Fe-rich abundance pattern (Zahedy et al., 2016a). If the ISM of massive galaxies at intermediate redshift is locally enriched by SNe Ia, then the observed $\mathrm{Fe} / \mathrm{Mg}$ is expected to decline with increasing projected distance.

To test this hypothesis, a sample of 13 massive quiescent galaxies (including two lensing galaxies) and 14 star-forming galaxies at intermediate redshifts $(\langle z\rangle \approx$ 0.5 ) has been assembled (Zahedy et al., 2016b). These galaxies were selected to have absorption spectra of background QSOs at $d \approx 10-400 \mathrm{kpc}$ available for constraining the radial profile of $\mathrm{Fe} / \mathrm{Mg}$. The right panel of Figure 6 displays the mean $\langle N(\mathrm{Fe}$ II $) / N(\mathrm{MgII})\rangle$ averaged over all individual components per halo versus $d$ for both star-forming galaxies (blue stars) and the quiescent galaxy population (red circles). Although the sample is still small, the distinction between quiescent and star-forming halos is already apparent in Figure 6. While the mean $\mathrm{Fe} / \mathrm{Mg}$ ratio is consistent with an $\alpha$-element enhanced pattern in the outer halo at $d>100 \mathrm{kpc}$ for both quiescent and star-forming galaxies, halo gas at $d<100 \mathrm{kpc}$ from quiescent galaxies exhibits an elevated iron abundance in comparison to star-forming galaxies.

The observed iron enrichment level in the inner halo of $z \approx 0.5$ quiescent galaxies is consistent with the solar value, similar to what is observed in the ISM of nearby elliptical galaxies (e.g. Mathews \& Brighenti, 2003; Humphrey \& Buote, 2006) and in the intracluster medium (e.g. de Plaa et al., 2007). The radial decline of the $\mathrm{Fe} / \mathrm{Mg}$ relative abundances supports the hypothesis that the gas is locally enriched by SNe Ia. The minimum fractional contribution of SNe Ia to the chemical enrichment in the inner halos of massive quiescent galaxies is found to be $f_{\mathrm{Ia}} \approx 15-20 \%$ based on the expected yields for Type Ia and core-collapse SNe (Iwamoto et al., 1999 ) and the observed $\mathrm{Fe} / \mathrm{Mg}$ ratio at $d<100 \mathrm{kpc}$.

\section{Quasar Host Halos}

In comparison to LRGs which are massive and quiescent, quasars powered by supermassive black holes also reside in massive halos of $M_{h} \gtrsim 3 \times 10^{12} \mathrm{M}_{\odot}$ (Porciani et al., 2004; White et al., 2012; Shen et al., 2013b) but in an active phase that lasts $\approx 10-100 \mathrm{Myr}$ (Martini, 2004). While the physical processes that drive the fueling and feedback of the central supermassive black holes are not well understood (e.g. Hopkins \& Hernquist, 2009; Heckman \& Best, 2014), quasar feedback is a critical ingredient in shutting down star formation in high-mass halos and producing redand-dead elliptical galaxies in all theoretical frameworks (e.g. Benson et al., 2003; Croton et al., 2006). Indeed, most nearby early-type galaxies are found to harbor a 
supermassive black hole at the center (Kormendy \& Richstone, 1995; Ho, 2008). Therefore, studying quasar hosts is an integral part of the effort to understand the growth of massive galaxies and observations of quasar host halos provide important clues for how the halo gas properties are shaped while the galaxies undergo an active quasar phase.
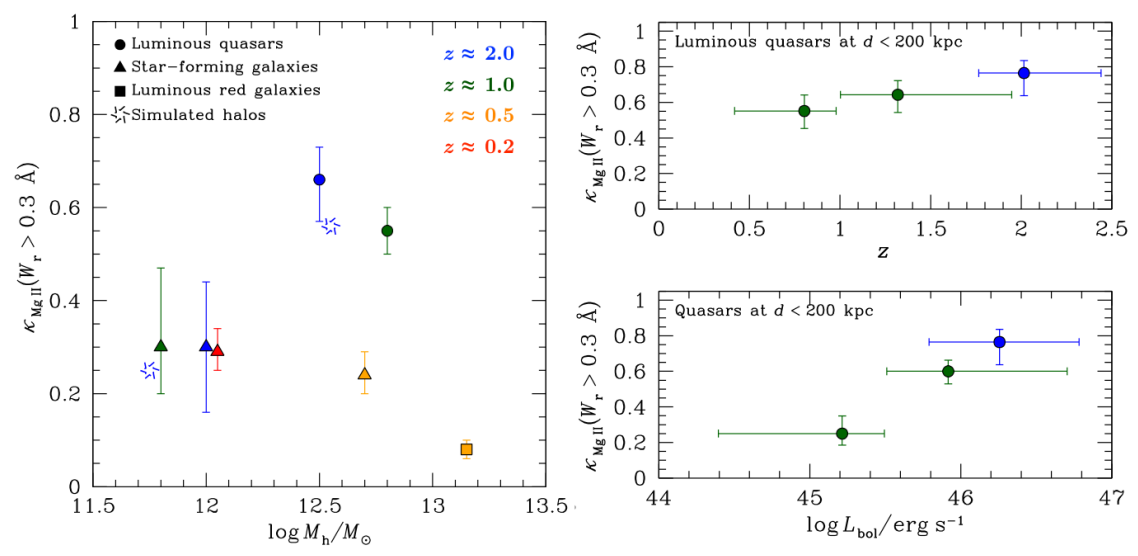

Fig. 7 Elevated cool gas covering fraction in quasar host halos. Left: Mean gas covering fraction $\kappa$ as a function of halo mass for galaxies and quasar hosts at different redshifts. The measurements for galaxies and quasars at $z<2$ are based on surveys of $\mathrm{Mg}$ II absorbers with $W_{r}(\mathrm{Mg}$ II $)>0.3$ $\AA$ and at $d<R_{\mathrm{vir}}$, where $R_{\mathrm{vir}}$ represents the virial radius of the host dark matter halos. Measurements for $z \approx 2$ star-forming galaxies (Rudie et al., 2012) and quasars (Prochaska et al., 2014) are inferred from the observed incidence of optically-thick H I absorbers and C II absorption observations, respectively. Closed triangles represent star-forming galaxies (Chen et al., 2010a; Lovegrove \& Simcoe, 2011; Rudie et al., 2012; Werk et al., 2014), squares represent quiescent LRGs (Huang et al., 2016), and circles represent quasar hosts (Prochaska et al., 2014; Johnson et al., 2015b). Star symbols are simulation predictions for $z \approx 2$ objects (Faucher-Giguère et al., 2016). Different colors indicate different redshift ranges of each dataset with red, orange, green, and blue corresponding to $z \approx 0.2,0.5,1.0$, and 2.0 , respectively. The mean gas covering fraction in quasar host halos is observed to be more than doubled from what is seen in star-forming galaxies at both low and high redshifts. Numerical simulations have attributed the elevated cool gas content in quasar hosts to either AGN feedback (Rahmati et al., 2015) or star formation feedback from neighboring satellite galaxies (Faucher-Giguère et al., 2016). Right: Mean gas covering fraction at $d<200$ $\mathrm{kpc}$ for quasar hosts at different redshifts (top) and with different bolometric luminosity (bottom). Colors denote different redshift ranges as defined in the left panel. While the mean gas covering fraction in quasar hosts is found to evolve only weakly with redshift, a steep correlation is observed between cool gas covering fraction and quasar luminosity. The elevated cool halo gas covering fraction observed in quasar hosts appears to be driven primarily by the most luminous quasar population. (Figure credit: Sean Johnson)

It has long been recognized that the intense radiation field from luminous quasars is sufficient to fully ionize their surrounding medium and to keep the IGM ionized over most of the cosmic history of the universe. Near the quasar redshifts, the incidence of $\operatorname{Ly} \alpha$ absorption lines in the quasar spectra is reduced due to an enhanced 
radiation field from the quasars in the proximity zone (e.g. Bajtlik et al., 1988). The line-of-sight quasar proximity effect, which is also observed in different ionic transitions such as Mg II and C IV, offers a unique means of constraining both the metagalactic ionizing radiation and the extent of the ionizing bubble local to the quasar (Dall'Aglio et al., 2008; Wild et al., 2008). However, a reduced incidence of absorption features is not observed along the transverse direction from quasars (e.g. Fernandez-Soto et al., 1995; Schirber et al., 2004). In addition, there also appears to be an excess of cool clouds in quasar halos (Bowen et al., 2006; Hennawi \& Prochaska, 2007; Farina et al., 2013, 2014; Prochaska et al., 2014).

The left panel of Figure 7 shows the mean covering fraction of cool halo gas $\kappa$ as a function of halo mass for galaxies (closed triangles and squares) and quasars (closed circles) at different redshifts. For comparison, expectations from numerical simulations are also included (open star symbols). Observations of halos around galaxies and quasars at $z<2$ are based on surveys of $\mathrm{Mg}$ II absorbers with $W_{r}(\mathrm{Mg} \mathrm{II})>0.3 \AA$ at projected distances less than the virial radius $R_{\mathrm{vir}}$ of the host dark matter halos. Observations of $z \approx 2$ star-forming galaxies (Rudie et al., 2012) and quasars (Prochaska et al., 2014) are inferred from the observed incidence of optically-thick HI absorbers and C II absorption features, respectively. While halos around normal galaxies display a roughly constant cool gas covering fraction of $\kappa \approx 30 \%$ for the star-forming population at all redshifts studied (e.g. Chen et al., 2010a; Lovegrove \& Simcoe, 2011; Rudie et al., 2012) and a suppressed cool gas content with $\kappa_{\sim}^{<} 10 \%$ for the quiescent population at $z \approx 0.5$ (e.g. Gauthier \& Chen, 2011; Huang et al., 2016), there appears to be a surge of cool gas with $\kappa \approx 60 \%$ in active quasar halos (Prochaska et al., 2014; Farina et al., 2013, 2014; Johnson et al., 2015a) at both low and high redshifts.

While state-of-the-art numerical simulations can reproduce the observed incidence of cool halo gas around star-forming galaxies and quasars at $z \approx 2$, the underlying physical mechanisms are fundamentally different. The elevated cool gas content in quasar hosts are attributed to either AGN feedback (Rahmati et al., 2015) or star formation feedback from neighboring satellite galaxies (Faucher-Giguère et al., 2016).

The observed line-of-sight proximity effect, together with an absence of transverse proximity effect, indicates that the quasar radiation field is not isotropic and that cool clouds outside of the radiation zone can still survive in the host halos. Understanding whether and how the incidence of cool gas varies with quasar properties provides further insights into the origin of the observed excess of cool halo gas around quasars. Using a sample of 195 projected quasar pairs separated by less than $300 \mathrm{kpc}$ in projected distance, it has been shown that the mean gas covering fraction (measured based on observations of $\mathrm{Mg}$ II absorption transitions) correlates strongly with the bolometric luminosity $L_{\mathrm{bol}}$ of the foreground quasar (Johnson et al., 2015a). Specifically, quasars that are ten times more luminous display on average 3-4 times higher cool gas covering fraction than low-luminosity quasars at $d<200 \mathrm{kpc}$ in the halos (lower-right panel of Figure 7). The elevated cool halo gas covering fraction observed in quasar hosts appears to be driven primarily by the most luminous quasars in the sample. 
The observed strong correlation between $\kappa$ and $L_{\text {bol }}$ is unlikely to be driven by an underlying mass dependence (c.f. Chen et al., 2010b), because the clustering amplitude of quasars remains roughly constant in different luminosity intervals (Shen et al., 2013b; Eftekharzadeh et al., 2015). The observed $L_{\text {bol }}$ dependence in cool halo gas covering fraction therefore has profound implications for the connection between halo gas on the $100 \mathrm{kpc}$ scale and quasar activities in the central parsec scale.

Possible explanations for the observed excess of cool gas along transverse sightlines include: (i) overdensity of galaxies in the quasar environment which is expected from the large clustering amplitude observed for luminous quasars, (ii) outflows from the quasar/nuclear starburst, and (iii) debris from galaxy interactions or mergers that trigger the luminous quasar phase. Of these scenarios, quasar outflows offer the most promising explanation but caveats remain. Significant contributions from correlated galaxies sharing the same large-scale overdensity peak can be ruled out based on the diminishing $\kappa$ observed at $d_{\sim} 200 \mathrm{kpc}$ from quasars (Johnson et al., 2015a). Merger remnants are unlikely to explain the observed cool gas at $d \sim 100$ $\mathrm{kpc}$, given that the quasar lifetime is shorter than the dynamical time.

Spatially extended outflows have been detected in [O III] emission out to $10-30$ kpc around luminous quasars at $z \sim 0.5$ with outflow velocities as high as $v_{\text {out }} \approx$ $1000 \mathrm{~km} \mathrm{~s}^{-1}$ (e.g. Greene et al., 2012; Liu et al., 2013, 2014). The observed spherical morphologies in these [O III] emitting nebulae suggest that outflows in these quasar hosts are not well-collimated. It is therefore possible that the absorbers detected at $d \sim 100 \mathrm{kpc}$ along transverse sightlines in quasar halos originate in these extended outflows with densities too low to be detected in emission.

An outflow origin also provides a natural explanation for the observed strong correlation between $\kappa$ and $L_{\text {bol }}$ with more luminous quasars driving higher mass outflow rates (e.g. Carniani et al., 2015). This is at least qualitatively consistent with the extreme kinematics displayed in some $(\approx 10 \%)$ quasar absorbers that spread over a velocity interval of $|\Delta v|_{\sim} 1000 \mathrm{~km} \mathrm{~s}^{-1}$ (e.g. Johnson et al., 2015a). However, free-expanding outflows, traveling at $v_{\text {out }}=1000 \mathrm{~km} \mathrm{~s}^{-1}$ at $15 \mathrm{kpc}$ from the quasar, are expected to reach $100 \mathrm{kpc}$ in $\approx 100 \mathrm{Myr}$ and longer if the outflows decelerate due to the gravitational potential of the host halo. With a typical quasar lifetime of $10-100 \mathrm{Myr}$ (e.g. Martini, 2004), the required outflow speed would need to exceed $1000 \mathrm{~km} \mathrm{~s}^{-1}$ in order for outflows near the peak of an active quasar phase to explain the observed excess of cool gas at $d \sim 100 \mathrm{kpc}$. With the energetics associated with fast-moving outflows, the gas is expected to be heated and highly ionized. If the cool clumps form within the hot outflows due to efficient cooling (e.g. Costa et al., 2015), then a strong correlation is expected between the incidence of cool gas and the presence of highly-ionized gas as probed by either [O III] emission (e.g. Greene et al., 2012) or O VI/C IV absorption (e.g. Grimes et al., 2009). Observations of highly-ionized gas associated with the low-ionization gas detected in quasar host halos will provide new insights into the origin of the $\kappa$ vs. $L_{\text {bol }}$ correlation.

Alternatively, the cool gas could originate in outflows from star-forming satellites as suggested by a recent simulation study (Faucher-Giguère et al., 2016). In this scenario, the distance required for the outflows to travel within the quasar life- 
time would be $\sim 10 \mathrm{kpc}$, rather than $\sim 100 \mathrm{kpc}$, and the required outflow energetics would be less extreme. However, the implication would be a more quiescent satellite environment around low-luminosity quasars in order to explain a reduced $\kappa$. Comparing the star formation histories of satellites around luminous and low-luminosity quasars will provide a necessary test for this scenario.

\section{Summary and Future Prospects}

Significant progress has been made over the last decade in characterizing the cool circumgalactic gas in massive halos of $M_{h}>10^{12} \mathrm{M}_{\odot}$ at $z \approx 0.2-2$ using absorption spectroscopy. This progress is facilitated by the unprecedentedly large galaxy and quasar samples available in the SDSS spectroscopic archive. Both massive galaxies and luminous quasars are rare. As a result, finding a background quasar in close projected distances for absorption-line studies of these rare objects requires a large survey volume. The large galaxy and quasar spectroscopic archive helps the assembly of statistically significant samples of close quasar and quiescent galaxy pairs and projected quasar pairs. These pair samples have enabled systematic studies of low-density gas beyond the nearby universe. Key findings from various studies can be summarized as follows:

1. Chemically-enriched cool gas of $T \sim 10^{4} \mathrm{~K}$ is present in massive quiescent halos at $z \sim 0.5$, with a declining annular average of covering fraction from $\langle\kappa\rangle \gtrsim 15 \%$ at $d<120 \mathrm{kpc}$ to $\langle\kappa\rangle \approx 5 \%$ out to the virial radius $R_{\mathrm{vir}}$. The improved statistics help rule out definitively an absence of cool gas in massive quiescent halos at $z \sim$ 0.5 , extending observations of cold gas in local early-type galaxies (e.g. Young et al., 2014) to those at intermediate redshifts.

2. Strong Mg II absorbers of $W_{r}(2796)>1 \AA$ produced by photo-ionized cool gas are not uncommon throughout quiescent halos from $d<100 \mathrm{kpc}$ to the virial radius $d \approx R_{\mathrm{vir}}$ and the observed $\mathrm{Mg}$ II absorbing strength in these halos does not depend on either galaxy luminosity or mass. The lack of correlation between $W_{r}(2796)$ and galaxy properties in quiescent halos suggests that the observed cool gas is likely to originate in infalling materials from the IGM, rather than outflowing gas from these early-type galaxies.

3. The velocity dispersion of $\mathrm{Mg}$ II absorbing gas around the majority $(\approx 90 \%)$ of massive quiescent galaxies is suppressed, at $\approx 60 \%$ of what is expected from the virial motion. Dissipation is expected if these $\mathrm{Mg}$ II absorbers originate in cool clumps condensed out of the hot halo through thermal instabilities and the clumps decelerate due to ram-pressure while moving through the hot halo. In this simple cloud model, the volume filling factor of the clumps is small in these massive halos with a mean number of $n_{\text {clump }} \sim 4$ per sightline in order to explain the large scatter found in the $W_{r}(2796)$ versus $d$ distribution, and a mean clump mass of $m_{\mathrm{cl}} \approx 5 \times 10^{4} \mathrm{M}_{\odot}$ in order to explain the suppression in velocity distribution.

4. While gas metallicity alone is insufficient for distinguishing between infalling and outflowing gas due to an unknown degree of chemical mixing in the CGM, 
the observed chemical composition of the gas offers important clues for the chemical enrichment history. The chemical composition of cool halo gas at $d_{\sim}<100 \mathrm{kpc}$ from massive quiescent galaxies displays an elevated iron abundance level that differs from an $\alpha$-element enhancement typically found in star-forming galaxies and in the IGM. The observed $\mathrm{Fe} / \mathrm{Mg}$ ratio implies a fractional contribution of SNe Ia to the total (Type Ia and core-collapse combined) of $f_{\mathrm{Ia}} \approx 15-20 \%$ in these inner massive halos. Beyond $d \approx 100 \mathrm{kpc}$, the observed $\mathrm{Fe} / \mathrm{Mg}$ ratio recovers the typical $\alpha$-element enhanced level.

5. There exists a strong correlation between the cool halo gas covering fraction $\kappa$ in quasar host halos and quasar bolometric luminosity $L_{\text {bol }}$, leading to a surge of cool gas in halos about luminous quasars at both low and high redshifts. The strong $\kappa-L_{\text {bol }}$ correlation suggests a physical link between cool gas content on scales of $100 \mathrm{kpc}$ and quasar activities on sub-parsec scales, but interpreting this strong correlation remains challenging. The primary difficulty lies in the relatively short quasar lifetime of $\approx 10-100$ Myr in comparison to the long dynamical time necessary to move gaseous clouds over a large distance of $\approx 200 \mathrm{kpc}$. Direct imaging of quasar-driven outflows and observations of highly-ionized gas associated with cool gas at $d \approx 200 \mathrm{kpc}$ are necessary to establish direct connections between outflows and cold gas detected at large distances.

Together, these findings suggest that infalling clouds from external sources are

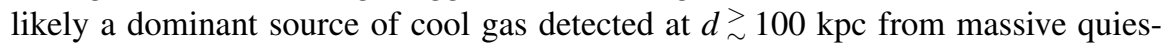
cent galaxies. The origin of the gas closer in is currently less certain, but SNe Ia driven winds appear to contribute significantly to cool gas found at $d<100 \mathrm{kpc}$.

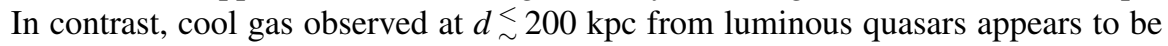
intimately connected to the on-going quasar activities. The observed strong correlation between cool gas covering fraction in quasar host halos and quasar bolometric luminosity remains a puzzle.

With new instruments and new survey data becoming available, continuing progress is expected in a number of areas over the next few years for a better understanding of the CGM in massive halos. In particular, spatially-resolved observations of quasar outflows in the inner $10-30 \mathrm{kpc}$ region, combined with absorption-line kinematics at $\sim 100 \mathrm{kpc}$ from the quasar, will provide key insights into the strong correlation between $\kappa$ and $L_{\text {bol }}$ in quasar host halos. Integral field unit (IFU) spectrographs available on large ground-based telescopes provide a powerful tool for imaging quasar outflows based on observations of high-ionization lines.

In addition, while measurements of chemical compositions provide a unique constraint for the physical origin of chemically-enriched gas in massive quiescent halos, measurements of $N(\mathrm{HI})$ are necessary for direct comparisons between observations and state-of-the-art cosmological simulations. The Cosmic Origins Spectrograph (COS; Green et al., 2012) on board the Hubble Space Telescope provides the spectral coverage necessary for $N(\mathrm{HI})$ measurements at $z_{\sim}^{<} 1$. With an increasing number of $z \approx 0.5$ LRGs found near the sightline of a UV bright QSO, there will soon be a statistical sample of massive quiescent halos with known $N(\mathrm{HI})$ at different projected distances for testing simulation predictions. 
Furthermore, understanding the roles of satellites and satellite interactions in producing chemically-enriched cool clumps in low-density halos requires deep galaxy survey data in quasar fields. With several wide-field integral field spectrographs being installed on ground-based telescopes, deep galaxy survey data in a large number of quasar fields will soon be available for systematic studies of the galaxy environments of kinematically complex absorbers.

Finally, little is known regarding the CGM properties and galactic environments of massive starburst galaxies with $M_{\text {star }} \gtrsim 10^{11} \mathrm{M}_{\odot}$ (e.g. Borthakur et al., 2013). Although these galaxies are very rare, contributing to roughly $10 \%$ of the massive galaxy population (with the rest being quiescent LRGs), they are intrinsically UV luminous and massive stars in these galaxies serve as the backlight for probing the internal star-forming ISM in front of the massive young stars. With numerous largescale galaxy surveys expected in the coming years (e.g. Zhu et al., 2015), combining intrinsic absorption-line observations with absorption spectroscopy along transverse sightlines (e.g. Rubin et al., 2010; Kacprzak et al., 2014) will be feasible for a statistically significant sample of massive starburst galaxies. These new data will offer an important empirical understanding of the impact of starbursts on the CGM in massive halos.

Acknowledgements The author wishes to thank Sean Johnson, Rebecca Pierce, Michael Rauch, and Fakhri Zahedy for providing helpful input and comments. In preparing this review, the author has made use of NASA's Astrophysics Data System Bibliographic Services.

\section{References}

Alexander D. M., Swinbank A. M., Smail I., et al. 2010, MNRAS, 402, 2211

Andrews H., Barrientos L. F., López S., et al. 2013, ApJ, 774, 40

Bajtlik S., Duncan R. C., Ostriker J. P., 1988, ApJ, 327, 570

Behroozi P. S., Conroy C., Wechsler R. H., 2010, ApJ, 717, 379

Belfiore F., Maiolino R., Maraston C., et al. 2016, MNRAS, 461, 3111

Benson A. J., Bower R. G., Frenk C. S., et al. 2003, ApJ, 599, 38

Bergeron J., Stasińska G., 1986, A\&A, 169, 1

Birnboim Y., Dekel A., 2003, MNRAS, 345, 349

Blake C., Collister A., Lahav O., 2008, MNRAS, 385, 1257

Blumenthal G. R., Faber S. M., Primack J. R., Rees M. J., 1984, Nature, 311, 517

Borthakur S., Heckman T., Strickland D., Wild V., Schiminovich D., 2013, ApJ, 768, 18

Bouché N., Murphy M. T., Péroux C., Csabai I., Wild V., 2006, MNRAS, 371, 495

Bowen D. V., Blades J. C., Pettini M., 1995, ApJ, 448, 634

Bowen D. V., Chelouche D., 2011, ApJ, 727, 47

Bowen D. V., Hennawi J. F., Ménard B., et al. 2006, ApJL, 645, L105

Bower R. G., Benson A. J., Malbon R., et al. 2006, MNRAS, 370, 645

Cantalupo S., Arrigoni-Battaia F., Prochaska J. X., et al. 2014, Nature, 506, 63

Carniani S., Marconi A., Maiolino R., et al. 2015, A\&A, 580, A102

Chen H.-W., Helsby J. E., Gauthier J.-R., et al. 2010a, ApJ, 714, 1521

Chen H.-W., Lanzetta K. M., Webb J. K., 2001a, ApJ, 556, 158

Chen H.-W., Lanzetta K. M., Webb J. K., Barcons X., 1998, ApJ, 498, 77

-, 2001b, ApJ, 559, 654 
Chen H.-W., Mulchaey J. S., 2009, ApJ, 701, 1219

Chen H.-W., Prochaska J. X., 2000, ApJL, 543, L9

Chen H.-W., Tinker J. L., 2008, ApJ, 687, 745

Chen H.-W., Wild V., Tinker J. L., Gauthier J.-R., et al. 2010b, ApJL, 724, L176

Chung A., van Gorkom J. H., Kenney J. D. P., Crowl H., Vollmer B., 2009, AJ, 138, 1741

Churchill C. W., Mellon R. R., Charlton J. C., et al. 2000, ApJ, 543, 577

Churchill C. W., Vogt S. S., Charlton J. C., 2003, AJ, 125, 98

Chynoweth K. M., Langston G. I., Yun M. S., et al. 2008, AJ, 135, 1983

Conroy C., van Dokkum P. G., Kravtsov A., 2015, ApJ, 803, 77

Costa T., Sijacki D., Haehnelt M. G., 2015, MNRAS, 448, L30

Crighton N. H. M., Hennawi J. F., Prochaska J. X., 2013, ApJL, 776, L18

Croton D. J., Springel V., White S. D. M., et al. 2006, MNRAS, 365, 11

Dall'Aglio A., Wisotzki L., Worseck G., 2008, A\&A, 491, 465

de Plaa J., Werner N., Bleeker J. A. M., et al. 2007, A\&A, 465, 345

Dekel A., Birnboim Y., 2006, MNRAS, 368, 2

Dekel A., Silk J., 1986, ApJ, 303, 39

Dessauges-Zavadsky M., Calura F., Prochaska J. X., et al. 2004, A\&A, 416, 79

Dubois Y., Gavazzi R., Peirani S., Silk J., 2013, MNRAS, 433, 3297

Eftekharzadeh S., Myers A. D., White M., et al. 2015, MNRAS, 453, 2779

Eisenstein D. J., Annis J., Gunn J. E., et al. 2001, AJ, 122, 2267

Eisenstein D. J., Hogg D. W., Fukugita M., et al. 2003, ApJ, 585, 694

Fabian A. C., 2012, ARA\&A, 50, 455

Farina E. P., Falomo R., Decarli R., et al. 2013, MNRAS, 429, 1267

Farina E. P., Falomo R., Scarpa R., et al. 2014, MNRAS, 441, 886

Faucher-Giguère C.-A., Feldmann R., Quataert E., et al. 2016, MNRAS, 461, L32

Faucher-Giguère C.-A., Hopkins P. F., Kereš D., et al. 2015, MNRAS, 449, 987

Faucher-Giguère C.-A., Kereš D., 2011, MNRAS, 412, L118

Ferland G. J., Porter R. L., van Hoof P. A. M., et al. 2013, Revista Mexicana de Astronoma y Astrofsica, 49, 137

Fernández X., Gim H. B., van Gorkom J. H., et al. 2016, ApJL, 824, L1

Fernandez-Soto A., Barcons X., Carballo R., Webb J. K., 1995, MNRAS, 277, 235

Ford A. B., Davé R., Oppenheimer B. D., et al. 2014, MNRAS, 444, 1260

Förster F., Schawinski K., 2008, MNRAS, 388, L74

Fox A., Richter P., Fechner C., 2014, A\&A, 572, A102

Fumagalli M., Hennawi J. F., Prochaska J. X., et al. 2014, ApJ, 780, 74

Fumagalli M., Prochaska J. X., Kasen D., et al. 2011, MNRAS, 418, 1796

Gallazzi A., Charlot S., Brinchmann J., et al. 2005, MNRAS, 362, 41

Gauthier J.-R., 2013, MNRAS, 432, 1444

Gauthier J.-R., Chen H.-W., 2011, MNRAS, 418, 2730

Gauthier J.-R., Chen H.-W., Cooksey K. L., et al. 2014, MNRAS, 439, 342

Gauthier J.-R., Chen H.-W., Tinker J. L., 2009, ApJ, 702, 50

-, 2010, ApJ, 716, 1263

Gilmore G., Wyse R. F. G., 1991, ApJL, 367, L55

Green J. C., Froning C. S., Osterman S., et al. 2012, ApJ, 744, 60

Greene J. E., Zakamska N. L., Smith P. S., 2012, ApJ, 746, 86

Grimes J. P., Heckman T., Aloisi A., et al. 2009, ApJS, 181, 272

Guo Q., White S., Li C., Boylan-Kolchin M., 2010, MNRAS, 404, 1111

Haardt F., Madau P., 2001, in Clusters of Galaxies and the High Redshift Universe Observed in $X$-rays, Neumann D. M., Tran J. T. V., eds.

Hamann F., 1997, ApJS, 109, 279

Hand N., Appel J. W., Battaglia N., et al. 2011, ApJ, 736, 39

Hansen S. M., Sheldon E. S., Wechsler R. H., Koester B. P., 2009, ApJ, 699, 1333

Hayes M., Melinder J., Östlin G., et al. 2016, ApJ, 828, 49 
Heckman T. M., 2002, in Astronomical Society of the Pacific Conference Series, Vol. 254, Extragalactic Gas at Low Redshift, Mulchaey J. S., Stocke J. T., eds., p. 292

Heckman T. M., Alexandroff R. M., Borthakur S., et al. 2015, ApJ, 809, 147

Heckman T. M., Best P. N., 2014, ARA\&A, 52, 589

Hennawi J. F., Prochaska J. X., 2007, ApJ, 655, 735

Ho L. C., 2008, ARA\&A, 46, 475

Hopkins P. F., Hernquist L., 2009, ApJ, 698, 1550

Huang Y.-H., Chen H.-W., Johnson S. D., Weiner B. J., 2016, MNRAS, 455, 1713

Humphrey P. J., Buote D. A., 2006, ApJ, 639, 136

Iwamoto K., Brachwitz F., Nomoto K., et al. 1999, ApJS, 125, 439

Johansson P. H., Naab T., Ostriker J. P., 2009, ApJL, 697, L38

Johnson S. D., Chen H.-W., Mulchaey J. S., 2013, MNRAS, 434, 1765

- 2015a, MNRAS, 452, 2553

-, 2015b, MNRAS, 449, 3263

Kacprzak G. G., Churchill C. W., Steidel C. C., Murphy M. T., 2008, AJ, 135, 922

Kacprzak G. G., Martin C. L., Bouché N., et al. 2014, ApJL, 792, L12

Kacprzak G. G., Murphy M. T., Churchill C. W., 2010, MNRAS, 406, 445

Kereš D., Katz N., Fardal M., et al. 2009, MNRAS, 395, 160

Kereš D., Katz N., Weinberg D. H., Davé R., 2005, MNRAS, 363, 2

Kirby E. N., Cohen J. G., Guhathakurta P., et al. 2013, ApJ, 779, 102

Kormendy J., Richstone D., 1995, ARA\&A, 33, 581

Kravtsov A. V., Borgani S., 2012, ARA\&A, 50, 353

Lanzetta K. M., Bowen D., 1990, ApJ, 357, 321

Lanzetta K. M., Bowen D. V., Tytler D., Webb J. K., 1995, ApJ, 442, 538

Larson R. B., 1974, MNRAS, 169, 229

Lee H., Skillman E. D., Cannon J. M., et al. 2006, ApJ, 647, 970

Lehner N., Howk J. C., Tripp T. M., et al. 2013, ApJ, 770, 138

Liang C. J., Chen H.-W., 2014, MNRAS, 445, 2061

Liu G., Zakamska N. L., Greene J. E., 2014, MNRAS, 442, 1303

Liu G., Zakamska N. L., Greene J. E., et al. 2013, MNRAS, 430, 2327

Lopez S., Barrientos L. F., Lira P., et al. 2008, ApJ, 679, 1144

Lovegrove E., Simcoe R. A., 2011, ApJ, 740, 30

Lundgren B. F., Brunner R. J., York D. G., et al. 2009, ApJ, 698, 819

Maiolino R., Gallerani S., Neri R., et al. 2012, MNRAS, 425, L66

Maller A. H., Bullock J. S., 2004, MNRAS, 355, 694

Mandelbaum R., Seljak U., Hirata C. M., 2008, JCAP, 8, 6

Marasco A., Crain R. A., Schaye J., et al. 2016, MNRAS, 461, 2630

Martini P., 2004, Coevolution of Black Holes and Galaxies, 169

Mathews W. G., Brighenti F., 2003, ARA\&A, 41, 191

Matteucci F., 2014, The Origin of the Galaxy and Local Group, Saas-Fee Advanced Course, Springer-Verlag Berlin Heidelberg, 37, 145

Ménard B., Wild V., Nestor D., et al. 2011, MNRAS, 417, 801

Mo H. J., Miralda-Escudé J., 1996, ApJ, 469, 589

Moster B. P., Somerville R. S., Maulbetsch C., et al. 2010, ApJ, 710, 903

Mulchaey J. S., 2000, ARA\&A, 38, 289

Mulchaey J. S., Chen H.-W., 2009, ApJL, 698, L46

Narayanan A., Charlton J. C., Misawa T., et al. 2008, ApJ, 689, 782

Nestor D. B., Johnson B. D., Wild V., et al. 2011, MNRAS, 412, 1559

Nestor D. B., Turnshek D. A., Rao S. M., Quider A. M., 2007, ApJ, 658, 185

Nomoto K., Tominaga N., Umeda H., et al. 2006, Nuclear Physics A, 777, 424

Oosterloo T., Morganti R., Crocker A., et al. 2010, MNRAS, 409, 500

Oosterloo T. A., Morganti R., Sadler E. M., et al. 2007, A\&A, 465, 787

Padmanabhan N., White M., Norberg P., Porciani C., 2009, MNRAS, 397, 1862

Peng Y.-j., Lilly S. J., Kovač K., et al. 2010, ApJ, 721, 193 
Petitjean P., Bergeron J., 1990, A\&A, 231, 309

Porciani C., Magliocchetti M., Norberg P., 2004, MNRAS, 355, 1010

Prescott M., Baldry I. K., James P. A., et al. 2011, MNRAS, 417, 1374

Prochaska J. X., Lau M. W., Hennawi J. F., 2014, ApJ, 796, 140

Rahmati A., Schaye J., Bower R. G., et al. 2015, MNRAS, 452, 2034

Rao S. M., Turnshek D. A., Nestor D. B., 2006, ApJ, 636, 610

Rauch M., Miralda-Escudé J., Sargent W. L. W., et al. 1997, ApJ, 489, 7

Rigby J. R., Charlton J. C., Churchill C. W., 2002, ApJ, 565, 743

Roseboom I. G., Pimbblet K. A., Drinkwater M. J., et al. 2006, MNRAS, 373, 349

Rubin K. H. R., Prochaska J. X., Koo D. C., Phillips A. C., Weiner B. J., 2010, ApJ, 712, 574

Rudie G. C., Steidel C. C., Trainor R. F., et al. 2012, ApJ, 750, 67

Salim S., Rich R. M., 2010, ApJL, 714, L290

Sarzi M., Falcón-Barroso J., Davies R. L., et al. 2006, MNRAS, 366, 1151

Sarzi M., Shields J. C., Schawinski K., et al. 2010, MNRAS, 402, 2187

Savage B. D., Sembach K. R., 1996, ARA\&A, 34, 279

Scalo J., Elmegreen B. G., 2004, ARA\&A, 42, 275

Schirber M., Miralda-Escudé J., McDonald P., 2004, ApJ, 610, 105

Serra P., Oosterloo T., Morganti R., et al. 2012, MNRAS, 422, 1835

Shankar F., Lapi A., Salucci P., De Zotti G., Danese L., 2006, ApJ, 643, 14

Sharma P., McCourt M., Quataert E., Parrish I. J., 2012, MNRAS, 420, 3174

Shen S., Madau P., Guedes J., Mayer L., Prochaska J. X., Wadsley J., 2013a, ApJ, 765, 89

Shen Y., McBride C. K., White M., et al. 2013b, ApJ, 778, 98

Singh R., van de Ven G., Jahnke K., et al. 2013, A\&A, 558, A43

Somerville R. S., Davé R., 2015, ARA\&A, 53, 51

Steidel C. C., Dickinson M., Meyer D. M., et al. 1997, ApJ, 480, 568

Steidel C. C., Dickinson M., Persson S. E., 1994, ApJL, 437, L75

Steidel C. C., Erb D. K., Shapley A. E., et al. 2010, ApJ, 717, 289

Steidel C. C., Kollmeier J. A., Shapley A. E., et al. 2002, ApJ, 570, 526

Stocke J. T., Keeney B. A., Danforth C. W., et al. 2014, ApJ, 791, 128

Sunyaev R. A., Zeldovich Y. B., 1970, Astrophysics and Space Science, 7, 3

Tal T., Wake D. A., van Dokkum P. G., 2012, ApJL, 751, L5

Thom C., Tumlinson J., Werk J. K., et al. 2012, ApJL, 758, L41

Tinker J. L., Chen H.-W., 2008, ApJ, 679, 1218

Tinker J. L., Leauthaud A., Bundy K., et al. 2013, ApJ, 778, 93

Tinsley B. M., 1979, ApJ, 229, 1046

Tojeiro R., Percival W. J., Heavens A. F., Jimenez R., 2011, MNRAS, 413, 434

Tolstoy E., Venn K. A., Shetrone M., et al. 2003, AJ, 125, 707

Tremonti C. A., Heckman T. M., Kauffmann G., et al. 2004, ApJ, 613, 898

Tsujimoto T., Nomoto K., Yoshii Y., et al. 1995, MNRAS, 277, 945

Tumlinson J., 2006, ApJ, 641, 1

Tumlinson J., Thom C., Werk J. K., et al. 2013, ApJ, 777, 59

Tumlinson J., Thom C., Werk J. K., et al. 2011, Science, 334, 948

Vale A., Ostriker J. P., 2004, MNRAS, 353, 189

Verdes-Montenegro L., Yun M. S., Williams B. A., et al. 2001, A\&A, 377, 812

Voit G. M., Donahue M., Bryan G. L., McDonald M., 2015, Nature, 519, 203

Werk J. K., Prochaska J. X., Thom C., et al. 2013, ApJS, 204, 17

Werk J. K., Prochaska J. X., Tumlinson J., et al. 2014, ApJ, 792, 8

White M., Myers A. D., Ross N. P., et al. 2012, MNRAS, 424, 933

White S. D. M., Rees M. J., 1978, MNRAS, 183, 341

Whiting M. T., Webster R. L., Francis P. J., 2006, MNRAS, 368, 341

Wild V., Kauffmann G., White S., et al. 2008, MNRAS, 388, 227

Wolfe A. M., Gawiser E., Prochaska J. X., 2005, ARA\&A, 43, 861

Yan R., Blanton M. R., 2012, ApJ, 747, 61

Yoon J. H., Putman M. E., Thom C., Chen H.-W., Bryan G. L., 2012, ApJ, 754, 84 
York D. G., Adelman J., Anderson Jr. J. E., et al. 2000, AJ, 120, 1579

Young L. M., Scott N., Serra P., et al. 2014, MNRAS, 444, 3408

Zahedy F. S., Chen H.-W., Rauch M., Wilson M. L., Zabludoff A., 2016a, MNRAS, 458, 2423

Zahedy F. S., Chen H.-W., Gauthier J.-R., Rauch M., 2016b, MNRAS, in press (arXiv:1611.09874)

Zheng Z., Coil A. L., Zehavi I., 2007, ApJ, 667, 760

Zhu G., Ménard B., Bizyaev D., et al. 2014, MNRAS, 439, 3139

Zhu G. B., Comparat J., Kneib J.-P., et al. 2015, ApJ, 815, 48

Zibetti S., Ménard B., Nestor D. B., et al. 2007, ApJ, 658, 161 